\title{
„E-INFRASTRUCTURES AUSTRIA PLUS“. KURZFASSUNG DES ABSCHLUSSBERICHTS ÜBER DAS HRSM-PROJEKT FÜR DAS SCHAFFEN, ANSIEDELN UND VERNETZEN VON INFRASTRUKTUR ZUM ERSTELLEN UND VERWALTEN VON PROJEKTANTRÄGEN UND FORSCHUNGSDATEN
}

\author{
von Thomas Haselwanter
}

Zusammenfassung: In den „Orientations towards the first Strategic Plan for Horizon Europe" “, dem Stategiepapier der EU-Kommission zum 9. EU-Forschungsrahmenprogramm findet sich zur Bedeutung von Open Science der folgende Satz: „Open science practices will be mainstreamed as the new modus operandi for EU research and innovation. "Open Science wird die Vorgabe, wie Forschung zukünftig mit Hilfe von digitalen Werkzeugen und Netzwerken getätigt werden soll. Die EU-Kommission ist hier zwar Vorreiter, lokale Fördergeber folgen dem Beispiel allerdings bereits. Für Forschungseinrichtungen bedeutet dies, dass die institutionelle Forschungsinfrastruktur weiterentwickelt werden muss um diesen Vorgaben zu genügen. Im Projekt "e-Infrastructures Austria Plus" wurden in sieben Arbeitspaketen damit begonnen Know-How und technische Infrastrukturen aufzubauen, die dafür benötigt werden.

Schlagwörter: eScience; Open Science; e-Infrastructures; Forschungsdatenmanagement

\section{“E-INFRASTRUCTURES AUSTRIA PLUS”. SHORT VERSION OF THE FINAL REPORT ON THE HRSM PROJECT FOR THE CREATION, ESTABLISHMENT AND NETWORIKING OF INFRASTRUCTURE FOR THE PREPARATION AND MANAGEMENT OF PROJECT APPLICATIONS AND RESEARCH DATA}

Abstract: In the "Orientations towards the first Strategic Plan for Horizin Europe",, the strategy paper of the EU-Commission on the 9th EU Framework Programme for Research and Innovation, the following sentence can be found on the importance of Open Science: "Open science practices will be mainstreamed as the new modus operandi for EU research and innovation. "Open Science will be the specification of how research should be carried out in the future with the help of digital tools and networks. Although the EU-Commission is a pioneer in this area, local funding bodies are already following suit. For research institutions, this means that the institutional research infrastructure must be further developed to meet these requirements. In the project "e-Infrastructures Austria Plus", seven work packages have begun to build up the know-how and technical infrastructures required for this. 
Keywords: eScience; Open Science; e-Infrastructures; Research Data Management

DOI: https://doi.org/10.31263/voebm.v73i1.3373

(C) Thomas Haselwanter

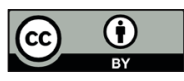

Dieses Werk ist - exkl. einzelner Logos und Abbildungen - lizenziert unter einer Creative-Commons-Lizenz Namensnennung 4.0 International

Dieser Artikel ist eine komprimierte Fassung des Endberichts zum Projekt. Mehr Informationen zu jedem Arbeitspaket finden sich im Endbericht (https://doi.org/10.25651/1.2020.0006).

\section{Einleitung}

Open Science beschleunigt die Forschung: Das ist einer der Hauptgründe von Fördergebern Open Science zu forcieren bzw. mittlerweile einzufordern. Die Entwicklung in Richtung Open Science findet über Landesgrenzen, über verschiedene Arten von Forschungseinrichtungen und über alle Fachbereiche hinweg statt. Sie hat Einfluss auf die Arbeitsweise der Forschenden und stellt neue Anforderungen an Forschungseinrichtungen. Die Hochschulraumstrukturmittelprojekte e-Infrastructures Austria (20142016) und e-Infrastructures Austria Plus (2017-2019) zielten darauf $a b$, diese neuen Anforderungen klarer zu erfassen und erste Infrastruktur umzusetzen. Dabei wird das „Open“ in Open Science auf verschiedene Weise interpretiert - auf der einen Seite geht es um Open Access und Open Data, also darum die wissenschaftlichen Publikationen und die Forschungsdaten eines Forschungsprojekts frei zugänglich zu machen. Auf der anderen Seite heißt „Open“ auch, dass sich die Forschung öffnet und zwar sowohl gegenüber anderen Forschenden und Studierenden, als auch gegenüber der interessierten Öffentlichkeit - Stichwort: Citizen Science. Im besten Fall entstehen durch die frei zugänglich gemachten Publikationen und Forschungsdaten neue kollaborative Forschungsprojekte und weitere Erkenntnisse. Gemein ist allen Definitionen, dass diese Änderungen im Wissenschaftsbetrieb durch eScience erreicht werden sollen. Als „eScience“ bezeichnet man Forschung, die kollaborativ mit Hilfe von digitaler Infrastruktur betrieben wird. Die Umsetzung von eScience ist ein komplexes Unterfangen. Die Arbeitspakete von e-Infrastructures Austria Plus thematisierten verschiedene Bereiche, die allesamt die Entwicklung von eScience unterstützen: 
- ein Research Lifecycle dient zur Veranschaulichung der einzelnen Schritte im Forschungsprozess und zur Darstellung von Diensten, die den Forschenden von der Universität zur Verfügung gestellt werden (Arbeitspaket Research Lifecycle)

- Policies zum Forschungsdatenmanagement klären nicht nur die Verantwortungen von Forschenden und Universitäten, sondern geben auch Kriterien für die Auswahl zu veröffentlichender Daten (Arbeitspaket Policy zum Forschungsdatenmanagement)

- Datenmanagementpläne vereinfachen die Nachnutzbarkeit durch die Vorgabe klarer Strukturen, verringern die Gefahr von Datenverlust und erhöhen die Chance, dass Daten und Dateiformate nach 10 Jahren noch lesbar sind (Arbeitspaket Datenmanagementpläne).

- Repositorien für Forschungsdaten sind die technische Infrastruktur, die für die Ablage und Veröffentlichung eigener Daten sowie zum Auffinden und Nachnutzung fremder Daten benötigt werden (Arbeitspaket Institutionelle Repositorien für Forschungsdaten).

- Ohne Beschreibung der Daten mittels Metadaten sind publizierte Forschungsdaten nicht auffindbar und können nicht nachgenutzt werden (Arbeitspaket Metadaten).

- Das Versehen der Forschungsdaten mit persistenten Identifikatoren ermöglicht eine dauerhafte Bewahrung und Nachnutzung von veröffentlichten Forschungsdaten. (Arbeitspaket Persistente Identifikatoren)

- GoFAIR stärkt die Kultur offener Forschungsdaten in der Gemeinschaft von Research Facilitators, Bibliotheken, politischen Entscheidungsträgern und Forschenden (Arbeitspaket GO FAIR). Alle genannten Inhalte fördern die Auffındbarkeit, Zugänglichkeit, Interoperabilität und Wiederverwendung von Forschungsdaten und tragen so zum Ökosystem eScience bei.

\section{Projektdaten}

Im Januar 2017 wurde das vom Bundesministerium für Bildung, Wissenschaft und Forschung (BMBWF) geförderte Hochschulraumstrukturmittelprojekt e-Infrastructures Austria Plus initiiert. Es war auf drei Jahre angelegt und lief bis Dezember 2019. Das Projekt hatte sich in Form von sieben Arbeitspaketen zum Ziel gesetzt, eine Roadmap für Forschungsdaten an den einzelnen Partnerinstitutionen zu erarbeiten. Ebenso standen technische Workflows und Lösungen für den Umgang mit Forschungsdaten und Qualitätsmanagement für Forschungsdatensysteme auf der Agenda. 


\subsection{Eckdaten}

Beginn: 1. Januar 2017 | Ende: 31. Dezember 2019

Projektmanagement: Universität Innsbruck

Projektpartner: neun Partnerinstitutionen

Website (Deutsch): https://www.e-infrastructures.at/de/

Website (Englisch): https://www.e-infrastructures.at/en/

Auftraggeber: Bundesministerium für Bildung, Wissenschaft und

Forschung (BMBWF)

Gesamtprojektkosten: EUR 1.933.070,- (davon Fördervolumen durch das BMWFW: EUR 300.000,-)

Projektkoordination: Universitäts- und Landesbibliothek Tirol, Universität Innsbruck

\subsection{Arbeitspakete}

\begin{tabular}{|c|c|c|}
\hline $\begin{array}{l}\text { Research Lifecycle: } \\
\text { Abbildung von } \\
\text { Forschungsprozessen }\end{array}$ & $\begin{array}{l}\text { Policy zum Forschungs- } \\
\text { datenmanagement: } \\
\text { Erarbeitung von Policies } \\
\text { zum FDM }\end{array}$ & $\begin{array}{l}\text { Datenmanagementpläne: } \\
\text { Aufbau lokaler } \\
\text { Kompetenzen zu DMPs }\end{array}$ \\
\hline $\begin{array}{l}\text { Institutionelle } \\
\text { Repositorien: Beispiel- } \\
\text { hafter Auswahlprozess } \\
\text { eines Repositoriums }\end{array}$ & $\begin{array}{l}\text { Metadaten: } \\
\text { Beschreibung von For- } \\
\text { schungsdaten im Sinne } \\
\text { der FAIR-Prinzipien }\end{array}$ & $\begin{array}{l}\text { Persistente } \\
\text { Identifikatoren: } \\
\text { Förderung der Zitierbar- } \\
\text { keit von Forschenden etc. }\end{array}$ \\
\hline $\begin{array}{c}\text { GO FAIR: } \\
\text { Linking Open Science in } \\
\text { Austria }\end{array}$ & \multicolumn{2}{|c|}{$\begin{array}{l}\text { Ursprünglich war auch ein Arbeitspaket zu Electronic } \\
\text { Lab Notebooks geplant, aber das Thema hatte sich } \\
\text { in Anbetracht der begrenzten Ressourcen als nicht } \\
\text { realisierbar herausgestellt }{ }^{2} \text {. }\end{array}$} \\
\hline
\end{tabular}




\subsection{Organisation und Partner}

E-Infrastructures Austria Plus zählte neun Projektpartner, acht öffentliche Universitäten und eine private:

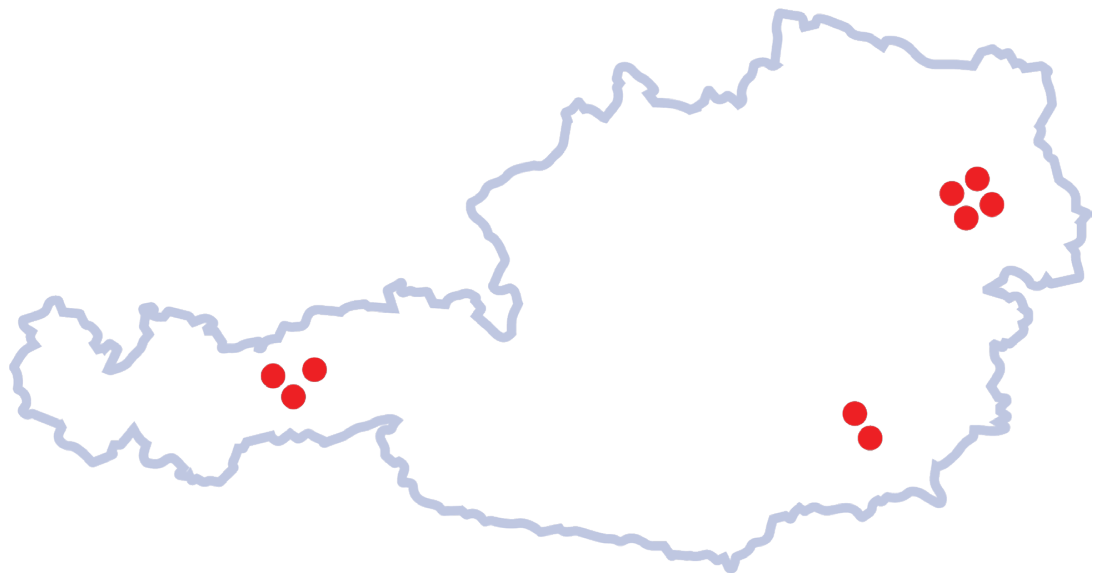

- Medizinische Universität Graz: vertreten durch Peter Schaffer, Leiter der Abteilung Forschungsdokumentation \& -evaluierung; Organisationseinheit Forschungsmanagement

- Medizinische Universität Innsbruck: vertreten durch Wolfram Rieneck, Internationale Projekte und Kooperationsprojekte; Forschungsservice und Innovation

- Medizinische Universität Wien: vertreten durch Susanne Friedl, Forschungsservice

- Technische Universität Wien: vertreten durch Beate Guba, Bibliotheksdirektorin und Barbara Sánchez Solís, Leitung des Zentrums für Forschungsdatenmanagement

- UMIT - Privatuniversität für Gesundheitswissenschaften Medizinische Informatik und Technik Hall in Tirol: vertreten durch Alexander Hoerbst, Head of the Research Division; Biomedical Computer Science and Mechatronics

- Universität Graz: vertreten durch Christian Kaier, Open Access Office \& Publikationsservices der Universitätsbibliothek

- Universität Innsbruck: vertreten durch Eva Ramminger, Leiterin der Universitäts- und Landesbibliothek Tirol und Thomas Haselwanter, Leiter der Abteilung Web- und Informationssysteme im Zentralen Informatikdienst 
- Universität Wien: vertreten durch Maria Seissl, Leiterin der Universitätsbibliothek und Paolo Budroni bis 31. August 2019, Leiter der Abteilung e-Infrastruktur, Universitätsbibliothek Wien, mittlerweile an die Technische Universität Wien gewechselt

Assoziierte Partner: Das Austrian Institute of Technology, die Freie Universität Bozen, das Know-Center der TU Graz, die Österreichische Akademie der Wissenschaften, das SBA-Research Center und die Universität für Angewandte Kunst Wien waren als assoziierte Partner am Projekt beteiligt.

\section{Research Lifecycle ${ }^{3}$}

Die Evolution der wissenschaftlichen Methoden hin zu eScience und Open Science transformiert den Forschungsprozess zu einem komplexen Workflow mit vielen Stakeholdern innerhalb der Forschungseinrichtungen. Die Darstellung in einem Research Lifecycle macht diesen Workflow nachvollziehbar, zeigt Verbindungen zwischen lokalen Stakeholdern auf und macht deren Zuständigkeiten sichtbar.

Es existieren bereits verschiedene Research Lifecycles, die an vielen Institutionen zu unterschiedlichen Zwecken in Verwendung sind. Zu Beginn der Arbeit an diesem Arbeitspaket wurde deshalb entschieden, vorhandene Research Lifecycles als Basis zu verwenden und diese mit Erkenntnissen aus Interviews mit Forschenden zu ergänzen.

\subsection{Interviews mit den Forschenden}

Insgesamt wurden 147 Leitfadeninterviews zum Forschungsdatenmanagement von vier Projektpartnern umgesetzt: Medizinische Universität Wien, Universität Innsbruck, Technische Universität Wien und der Wirtschaftsuniversität Wien. Aus den Interviews sollten Anliegen an einen effizienten und nachhaltigen Umgang mit Daten extrahiert und der Wissensstand der Wissenschaftler zu aktuellen Entwicklungen erhoben werden. Ursprünglich sollten auch sogenannte Research Lifecycle Patterns entstehen, mittels derer die Arbeitsweise in verschiedenen Fachbereichen im Research Lifecycle abgebildet werden sollte. Der Leitfaden ${ }^{4}$ enthielt u. a. Fragen zu folgenden Themen:

- Informationen zu den eigenen Forschungsdaten

- Umgang mit Forschungsdaten (u.a. speichern, verwalten, archivieren, Datenverlust) 
- Veröffentlichen und Teilen von Forschungsdaten (u.a. Lizenzen, Verträge, Datenschutz, Fremddienstleister)

- Bekanntheit von „Repository“, „Forschungsdatenmanagement", „Datenmanagementplan“, „FAIR-Prinzipien“

Im Laufe der Befragung zeigte sich, dass sich Daten, Methoden und Vorgehensweisen sowohl von Fachbereich zu Fachbereich wie auch von Forschenden zu Forschenden so sehr unterscheiden, dass eine Abbildung von standardisierten Prozessen nicht realisierbar war. Jedoch gab die offene Methode der Datenerhebung Raum für neu entstandene Bedürfnisse seitens der Forschenden durch die Anforderungen von außen. Die Anliegen wurden identifiziert und an die entsprechenden Leitungsorgane und Stakeholder (z. B. Vizerektorate, Zentraler Informatikdienst) kommuniziert. Universitätsübergreifend wurden folgende Anliegen identifiziert: Bedarf einer Open Science-Anlaufstelle, Bereitstellung entsprechender Speichersysteme (u.a. Repositorien für Forschungsdaten) und Förderung der Kultur des Data Stewardship.

Neben der Identifizierung und Kommunikation der Bedürfnisse trugen die Interviews zum Aufbau von Kontakten zu den Forschenden und zur Sensibilisierung verschiedener Stakeholder auf unterschiedlichen Ebenen zum Thema Forschungsdatenmanagement bei. Darüber hinaus ergänzten sie bereits bestehende Research Lifecycles

\subsection{Research Lifecycle}

Bei der Untersuchung der verschiedenen bereits existierenden Lifecycles wurde sichtbar, dass einige unter dem Begriff Research Lifecycle den gesamten Forschungsprozess abbilden, andere aber nur den Umgang mit Daten dokumentieren und deshalb eigentlich Research Data Lifecycle genannt werden müssten.

Schließlich wurde der Research Lifecycles der University of Central Florida $^{5}$ als Basis gewählt und um Inhalte des Research Data Lifecycles des UK Data Services ${ }^{6}$ ergänzt. Weitere Ergänzungen ergaben sich und aus den Interviews mit den Forschenden.

Der so entstandene allgemeine Research Lifecycle weist einige Knotenpunkte mehr auf als der ursprüngliche. Im Planungszyklus wurde verstärkt Fokus auf die Planung des Forschungsvorhabens gelegt und auch notwendige Werkzeuge und neue Anforderungen der Fördergeber wurden abgebildet. Der Projektzyklus wurde mit Tätigkeiten aus dem Data Lifecycle vervollständigt (z.B. die Erfassung und Analyse der Daten). Sofern an einer 
Institution Data Stewards eingerichtet wurden, können sie ebenfalls im Research Lifecycle gefunden werden. Auch der Publikationszyklus wurde ergänzt. Neben rechtlicher Beratung zu einigen Themen findet sich hier ein zusätzlicher innerer Zyklus, der inhaltlich an Förderplanung und Fördermanagement der vorigen Zyklen anschließt. Ohne persistente Identifikatoren (PIDs) werden Forschungsergebnisse künftig nicht mehr sichtbar werden. Die Sichtbarkeit kann zusätzlich durch die Wahl eines angesehenen Repositoriums erhöht werden. Die Anpassungen beim Digital Scholarship-Zyklus hielten sich dagegen in Grenzen. Neben dem auch hier unerlässlichen PID-Management wurde lediglich der Zyklus ergänzt, der die Ergebnisse eines Forschungsvorhabens betrifft.

\subsection{Ergebnisse}

Der für die Universität Innsbruck erstellte Forschungsprozess, kann als Vorlage für andere Universitäten dienen. Der Research Lifecycle wurde als Vektorgrafik (SVG) erstellt und ist dadurch einfach skalierbar und anpassbar. Zusätzlich wurden Links bei allen Knotenpunkten und Zyklen hinterlegt, sodass die Grafik oder Teile davon als verweissensitive Grafik (Imagemap) im Internet verwendet werden kann. Für die Erstellung einer TemplateWebsite, die diese Links unterstützt, stehen Markdown Dateien zur Verfügung. Alle Deliverables stehen auf der Website www.e-infrastructures.at zum Download bereit oder sind auf Nachfrage erhältlich.

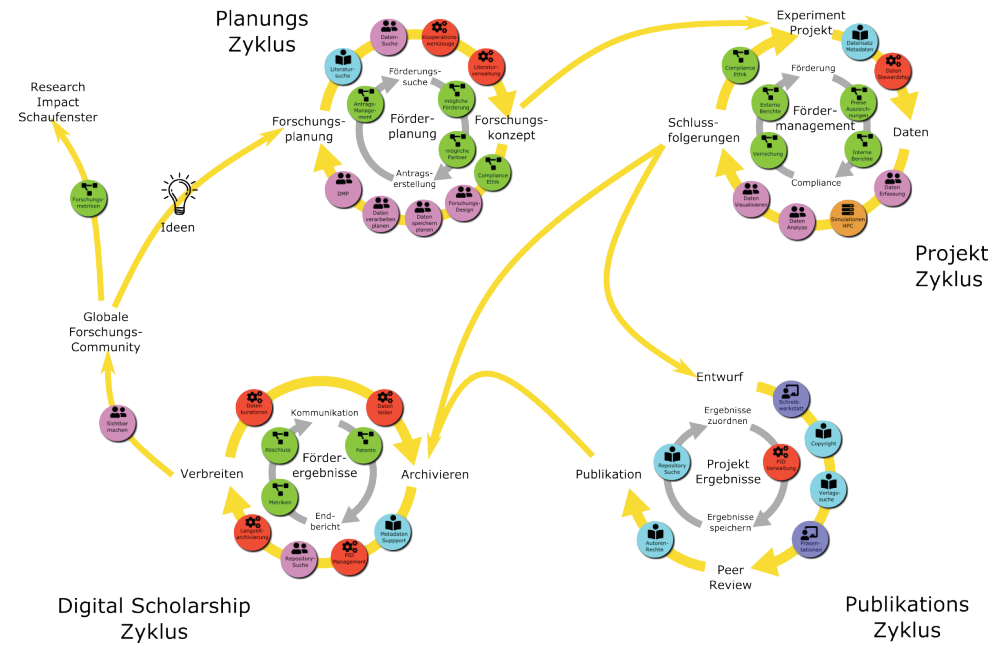

Abb. 1: Research Lifecycle der Universität Innsbruck ${ }^{7}$ 


\subsection{Empfehlungen \& Bedürfnisse}

- Research Lifecycle zur zentralen Dokumentation

Der Research Lifecycle kann als zentraler Startpunkt der gesamten Dokumentation zum Forschungsprozess dienen. Die Abbildung fördert die Orientierung der Wissenschaftler, zeigt Anlaufstellen zu verschiedenen Schritten im Forschungsprozess und verdeutlicht allen Beteiligten ihre Rollen und Verantwortlichkeiten.

- eScience Portfolio Management

Ein Research Lifecycle kann die Weiterentwicklung der eScience-Infrastruktur innerhalb einer Institution mit verschiedenen Stakeholdern steuern.

- Einrichtung einer Open-Science-Anlaufstelle

Eine zentrale Anlaufstelle zu Open Science kann Informationsmaßnahmen zu Open Science und FAIR-Prinzipien umsetzen und (zukünftige) Forschende zum Forschungsdatenmanagement beraten und schulen. Darüber hinaus kann sie bei der Erstellung von Datenmanagementplänen und bei rechtlichen Fragen zu Weitergabe, Lizenzierung und Urheberrechten von Forschungsdaten unterstützen.

\section{Policy für Forschungsdatenmanagement ${ }^{8}$}

Jede Forschungseinrichtung sollte über eine Forschungsdatenmanagement-Policy (FDM-Policy) verfügen, in der Regelungen für die Kuratierung und das Management von Forschungsdaten definiert sind. Eine FDM-Policy enthält dabei immer Regelungen für alle Stakeholder einer Forschungseinrichtung. Die Policy definiert Regelungen zum Umgang mit Forschungsdaten für die Wissenschaftlerlnnen, und sie enthält Angaben darüber, welche Voraussetzungen eine Forschungseinrichtung dafür zur Verfügung stellt.

Ziel der Arbeit im Arbeitspaket war die Entwicklung von FDM Policies, sowie die Schulung von heterogenen Gruppen von Stakeholdern, die anschließend an ihren Einrichtungen die FDM Policy umsetzen. Zielgruppen waren Vizerektoren, Forschungsservices, Bibliotheken, IT-Services, Betriebsräte, Rechtsabteilungen, Vertreter einzelner Disziplinen. Mit dem Ziel der Implementierung von Policies in Österreich wurde auf Basis der Ergebnisse des Projekts LEARN ${ }^{9}$ eine Muster-Policy entworfen und österreichweit 21 Workshops durchgeführt, die dazu beitrugen, dass fünf österreichische Universitäten eine Policy veröffentlicht haben und weitere acht 
Universitäten daran arbeiten oder unmittelbar vor der Implementierung stehen.

Die verabschiedeten Policies der Universitäten und Forschungseinrichtungen:

- Universität für Musik und darstellende Kunst Wien (2017) ${ }^{10}$

- Medizinische Universität Wien (2018) ${ }^{11}$

- Technische Universität Wien (2018) ${ }^{12}$

- Universität Graz (2019) ${ }^{13}$

- Wirtschaftsuniversität Wien (2019) ${ }^{14}$

An anderen neun Forschungseinrichtungen sind derartige Regelungen in Arbeit. Aktuelle Informationen über veröffentlichte Policies finden sich auf forschungsdaten.info ${ }^{15}$. Außerhalb des Projekts wurde diese Musterpolicy auch international als konkrete Richtlinie realisiert, wie u.a. Università degli Studi di Milano ${ }^{16}$, Università di Padova ${ }^{17}$.

\subsection{Empfehlungen \& Bedürfnisse}

- Compliance

Eine FDM-Policy, die die Regeln zum Forschungsdatenmanagement an einer Institution verankert und sie transparent macht, trägt zum Wandel in Richtung eines aktiven Umgangs mit Forschungsdaten bei.

- Einrichtung der Services vor oder mit der Verabschiedung der FDM-Policy Die Verabschiedung der Policy ist sinnvoll, wenn bereits entsprechende Services zum Forschungsdatenmanagement von der Einrichtung bereitgestellt werden.

\section{Datenmanagementpläne ${ }^{18}$}

\subsection{Vorgaben der Fördergeber}

Bereits im Vorgängerprojekt e-Infrastructures Austria (2014-2016) entstanden Templates zu Datenmanagementplänen als eine Möglichkeit zur Steuerung des Speicherns von Forschungsdaten. Ein kritischer Baustein im Forschungsdatenmanagement wurden sie aber erst durch die Vorgaben internationaler und nationaler Fördergeber. Die Europäische Kommission stellte klar, dass ab dem Förderprogramm Horizon 2020 DMPs verpflichtend zu erstellen sind und nahm zusätzlich die FAIR-Prinzipien in die DMPVorgaben mit auf. 
Im Januar 2019 führte der FWF als größte österreichische Förderorganisation eine Open-Access-Policy für Forschungsdaten ein. Der FWF verlangt darin für alle Projekte, die nach dem 01.01.2019 nach den neuen Antragsrichtlinien bewilligt werden, einen DMP. Die DMP-Vorlage ${ }^{19}$ des FWF steht im Einklang mit den „Core Requirements for Data Management Plans ${ }^{20 "}$ von Science Europe.

Von Seiten des FFG und des WWTF liegen zwar noch keine Pläne für die flächendeckende Einführung von DMPs vor, beide nehmen aber zum Umgang mit Forschungsdaten Stellung.

Der FFG beschreibt DMPs als gutes Tool, um sich mit Forschungsdatenmanagement auseinanderzusetzen. Eine flächendeckende Einführung von DMPs ist dennoch nicht geplant, für OpenScience-Initiativen und Pilotprojekte in einzelnen Fachbereichen aber sehr wohl.

Der WWTF legt großen Wert auf interdisziplinäre und kollaborative Forschung, um Forschende unterschiedlicher Fakultäten und Institutionen zusammenzubringen und interaktive Partnerschaften in Wien zu fördern. Explizite Planung zum Forschungsdatenmanagement ist derzeit in den Lebenswissenschaften, besonders in den klinischen Calls, verpflichtend vorgesehen, DMPs werden aber noch nicht verlangt.

\subsection{Know-how-Aufbau}

Sowohl die Pläne der Europäischen Kommission als auch des FWF waren bereits zu Projektstart bekannt. Anfang 2018 begann deshalb nach einem Abstimmungstreffen mit dem FWF der Know-how-Aufbau in den am Projekt beteiligten Institutionen. Von Seiten des FWF wurde der Wunsch geäußert, dass die österreichischen Forschungsinstitutionen klar erkennbare Ansprechpersonen zu DMPs etablieren sollten. Um diese Personen zu unterstützen wurden Informationen über DMP-Beispiele gesammelt. So hat die Association of European Research Libraries (LIBER) einen Katalog mit DMP-Beispielen ${ }^{21}$ aus unterschiedlichen Disziplinen publiziert.

Auf DMPOnline finden sich unterschiedliche DMP-Templates von europäischen Fördergebern ${ }^{22}$.

\subsection{Disziplinspezifischer DMP für die Sozialwissenschaften}

Eine weitere Aufgabe die sich die Arbeitsgruppe gestellt hat, war die Anpassung des FWF-Template an eine Forschungsdisziplin. Mitarbeiter des Austrian Social Science Data Archive (AUSSDA) und der Wirtschaftsuniversität Wien setzten dieses Vorhaben für die Sozialwissenschaften ${ }^{23}$ um. 
Sie entwickelten ein Template mit besonderem Fokus auf quantitative Daten. Ursprünglich wurde versucht, nicht zu weit von der Grundstruktur des FWF-Templates abzuweichen, letztlich wurde es um die Sektionen „Administrative Data“ und Fragen zum Ressourcenaufwand erweitert. Zur Anwendung des disziplinspezifischen Templates für die Sozialwissenschaften gab es bislang noch wenig Rückmeldung.

\subsection{Vergleich und Bewertung ausgewählter DMP-Tools}

\begin{tabular}{|c|c|c|c|}
\hline & RDMO & DMPROADMAP & $\begin{array}{l}\text { DATA STEWARD- } \\
\text { SHIP WIZARD }\end{array}$ \\
\hline Website & $\begin{array}{l}\text { https://rdmorganiser. } \\
\text { github.io/ }\end{array}$ & $\begin{array}{l}\text { https://github.com/ } \\
\text { DMPRoadmap/road- } \\
\text { map }\end{array}$ & https://ds-wizard.org/ \\
\hline Dokumentation & $\begin{array}{l}\text { https://rdmorganiser. } \\
\text { github.io/dokumen- } \\
\text { tation/ }\end{array}$ & $\begin{array}{l}\text { https://github.com/ } \\
\text { DMPRoadmap/road- } \\
\text { map/wiki/Installation }\end{array}$ & $\begin{array}{l}\text { https://docs.ds- } \\
\text { wizard.org/ }\end{array}$ \\
\hline Use Cases & \multicolumn{2}{|c|}{$\begin{array}{l}\text { User erstellen ein „Projekt“. Dort werden } \\
\text { „Tasks“ angelegt, die User und Betreuer abar- } \\
\text { beiten. DMPs können auf Basis von Templates } \\
\text { erstellt werden. Das Ergebnis für ein DMP ist } \\
\text { ein Dokument. } \\
\text { User erstellen einen DMP auf Basis von Tem- } \\
\text { plates. Als Ergebnis wird ein Dokument erstellt. }\end{array}$} & $\begin{array}{l}\text { Auf Basis von „Know- } \\
\text { ledge Models“ können } \\
\text { Questionnaires aus- } \\
\text { gefüllt werden. Das } \\
\text { können Checklisten } \\
\text { oder DMPs sein, die } \\
\text { dann in verschiedene } \\
\text { Formate exportiert } \\
\text { werden können. }\end{array}$ \\
\hline $\begin{array}{l}\text { Ergänzungen während } \\
\text { des Projekts („leben- } \\
\text { diger“ DMP) }\end{array}$ & $\begin{array}{l}\text { Nein, nur neuer DMP } \\
\text { möglich }\end{array}$ & $\begin{array}{l}\text { Nein, nur neuer DMP } \\
\text { möglich }\end{array}$ & $\begin{array}{l}\text { Teilweise } \\
\text { (Questionnaire kann } \\
\text { unterbrochen werden) }\end{array}$ \\
\hline $\begin{array}{l}\text { Erstellung von Tem- } \\
\text { plates }\end{array}$ & XML & $\begin{array}{l}\text { Über Administrations- } \\
\text { menü erstellen }\end{array}$ & $\begin{array}{l}\text { Über Knowledge- } \\
\text { Model Editor }\end{array}$ \\
\hline DMP Export/Import & $\mathrm{XML}$ & $\mathrm{Ja}$ & $\mathrm{Ja}$ \\
\hline DMP Exportformate & $\begin{array}{l}\text { PDF, Rich Text For- } \\
\text { mat, OpenOffice, } \\
\text { Microsoft Office, } \\
\text { HTML, Markdown, } \\
\text { mediawiki, LaTeX }\end{array}$ & $\begin{array}{l}\text { csv, HTML, PDF, text, } \\
\text { docx }\end{array}$ & $\begin{array}{l}\text { PDF, HTML, Micro- } \\
\text { soft Office, OpenOf- } \\
\text { fice, JSON }\end{array}$ \\
\hline Verfügbare Sprachen & deutsch und englisch & $\begin{array}{l}\text { deutsch, englisch, } \\
\text { französisch, } \\
\text { portugiesisch }\end{array}$ & englisch \\
\hline getestete Version & $\begin{array}{l}\text { Januar } 2019- \\
\text { Version } 0.12 .0\end{array}$ & $\begin{array}{l}\text { Dezember } 2018 \text { - } \\
\text { Version 2.1.1 }\end{array}$ & $\begin{array}{l}\text { keine Versionen, } \\
\text { Tests bis Juli } 2019 \\
\text { abgeschlossen }\end{array}$ \\
\hline
\end{tabular}

Tab. 1: Vergleich der DMP-Tools (siehe: https://doi.org/10.25651/1.2019.0007) 
Die Universität Innsbruck stellte einen Vergleich zwischen den drei OnlineTools Research Data Management Organizer (RDMO), DMPRoadmap und Data Stewardship Wizard an. Der Vergleich bezog sich auf die Bereiche Anwendung, Integration, Sicherheit, Rechte und Weiterentwicklung. Die getesteten Tools arbeiten mit unterschiedlichen Ansätzen zur Erstellung von DMPs. Während RDMO und DMPRoadmap DMPs auf Projektebene erstellen, basiert der Data Stewardship Wizard auf allgemein anerkannten Modellen der Wissensgenerierung (Knowledge Models).

\section{Anwendung}

Bei RDMO und DMPRoadmap ist keine Versionierung möglich, d.h. ein „lebendiger DMP“, wie zum Beispiel bei Horizon 2020 gefordert, ist nur bedingt umsetzbar. User können dies nur über einen Export des Dokuments und einen erneuten Import der Datei realisieren, was in der konkreten Anwendung zu Problemen führen könnte, da in der Regel möglichst intuitiv zu bedienende Vorgehensweisen erwartet werden. Beim Data Stewardship Wizard lässt sich eine Versionierung teilweise umsetzen, indem man den Questionnaire unterbrechen kann. Im Output wird entsprechend vermerkt, dass nicht alle Fragen beantwortet wurden. Allerdings schlug der Versuch fehl, den Plan als JSON zu exportieren und wieder zu importieren.

\section{Integration}

Gegenwärtig noch ausständig ist die Integration persistenter Identifikatoren, wie ORCID und Digital Object Identifiers (DOI). DMPRoadmap sieht dies als Idee für die Zukunft. Alle getesteten Systeme besitzen derzeit nur minimale APIs. Dadurch gibt es aktuell keine Möglichkeit zur Integration dieser Tools mit lokalen, bestehenden Systemen. Über die API lassen sich beim RDMO Informationen zu Projekten oder Plänen abrufen, bei DMPRoadmap kann zusätzlich ein DMP auf Basis existierender Templates erstellt werden (ohne befüllte Daten). Der Data Stewardship Wizard hat das größte Potenzial, da es sich in einen Webservice integrieren lässt.

\section{Rechte und Sicherheit}

In den Tools lassen sich verschiedene Rollen mit unterschiedlichen Berechtigungen vergeben. Die Rechte bzw. Rollen ähneln sich in den verschiedenen Tools. Allerdings lässt sich die Rechtevergabe nicht zentral steuern. Dies führt zu einer aufwendigen händischen Rechtevergabe. 


\section{Weiterentwicklung}

Hinsichtlich der Weiterentwicklung sticht der Data Stewardship Wizard als Teil der „Machine-Actionable-DMP“- Initiative hervor, allerdings schränkt die Programmiersprache Haskell den Entwicklerkreis sehr ein.

\section{Fazit}

Insgesamt zeigte sich, dass die Tools für die Erstellung eines DMPs zur einmaligen Verwendung geeignet sind, jedoch sind sie nicht mit bestehenden internen oder externen Systemen verknüpfbar, ermöglichen keine Versionierung eines „lebendigen“ DMPs und/oder bieten keine zentrale Steuerung der Rechteverwaltung. Die Tools sind begrenzt hilfreich, jedoch nicht nachhaltig. Darüber hinaus wird zum Ausfüllen der DMPs weiterhin fachliche bzw. institutionelle Unterstützung benötigt. Aufgrund der nicht ausreichenden existierenden Tools wird an der Technischen Universität Wien an einem Tool für „machine-actionable DMPs“ gearbeitet.

\subsection{Machine-actionable DMPs und automatisiertes DMP-Tool}

Die Technische Universität Wien erstellt einen Piloten (Proof of Concept) für ein automatisiertes DMP-Tool ${ }^{24}$. Ziel ist es, den manuellen Aufwand für die Forschenden zu minimieren und gleichzeitig die Qualität der bereitgestellten Informationen zu erhalten. Die Lösung basiert auf machineactionable DMPs, den DMPs der zukünftigen Generation. Ziele eines automatisierten, institutionell eingebetteten DMP-Tools:

- Steigerung der Qualität von DMPs, z.B. Automatisierung der DMPValidierung

- Erleichterung des Prozesses für Forschende, z.B. direkt auf Unterstützung anderer institutioneller Stakeholder zugreifen

- Vermeidung von Doppelarbeit, z.B. Publikationen nur einmal eintragen

- Nutzung der institutionellen Infrastruktur wird gefördert, z.B. Datenrepositorium und Helpdesk

Dieses Konzept integriert TU Wien-interne Systeme (vorhandene und zu entwickelnde) so weit, dass das DMP-Tool mit diesen Systemen kommunizieren kann (interne Projektdatenbanken, CRIS-Systeme, Adress-Datenbanken etc.), um wirklich „machine-to-machine actionable“ zu sein. 
Die folgende Abbildung stellt einen Workflow zum automatisierten Datenmanagement dar, wie er vom automatisierten DMP-Tool umgesetzt werden soll.
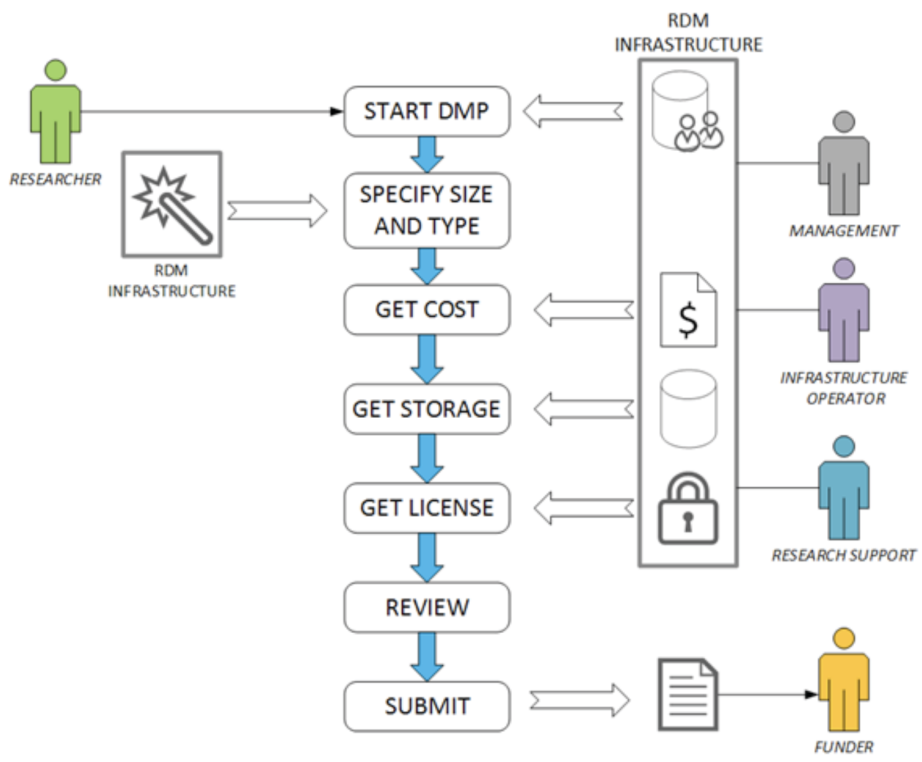

Abb. 2: Tomasz Miksa, Automated Data Management Workflow

Im Artikel „Ten principles for machine-actionable data management plans ${ }^{25 " ~ s i n d ~ d i e ~ G r u n d s a ̈ t z e ~ d e r ~ m a s c h i n e l l ~ v e r a r b e i t b a r e n ~ D M P s ~ u ̈ b e r-~}$ sichtlich dargestellt. Weitere Informationen zu machine-actionable DMPs finden sich auf der Website der Research Data Alliance (RDA) ${ }^{26}$. Ziel der Working Group ist es, machine-actionable DMP-Modelle unabhängig von spezifischen Templates von Fördergebern zu entwickeln. Um diese Vision zu erreichen, wird ein gemeinsames Datenmodell mit einem Kernsatz von Elementen entwickelt und Referenzimplementierungen des Datenmodells unter Verwendung gängiger Formate wie JSON, XML, RDF, etc. zur Verfügung gestellt.

Im Folgenden wird ein BPMN Prozess für machine-actionable DMPs dargestellt. Prozesse helfen bei der Identifizierung von:

- Aufgaben der unterschiedlichen Stakeholder, z.B. Bereitstellung von Speicherkosten von ICT-Services

- erforderlichen Systemen, z.B. Repositorien, Kalkulationsservices etc.

- notwendigen Konzepten, z.B. Kostenmodelle für Storage 


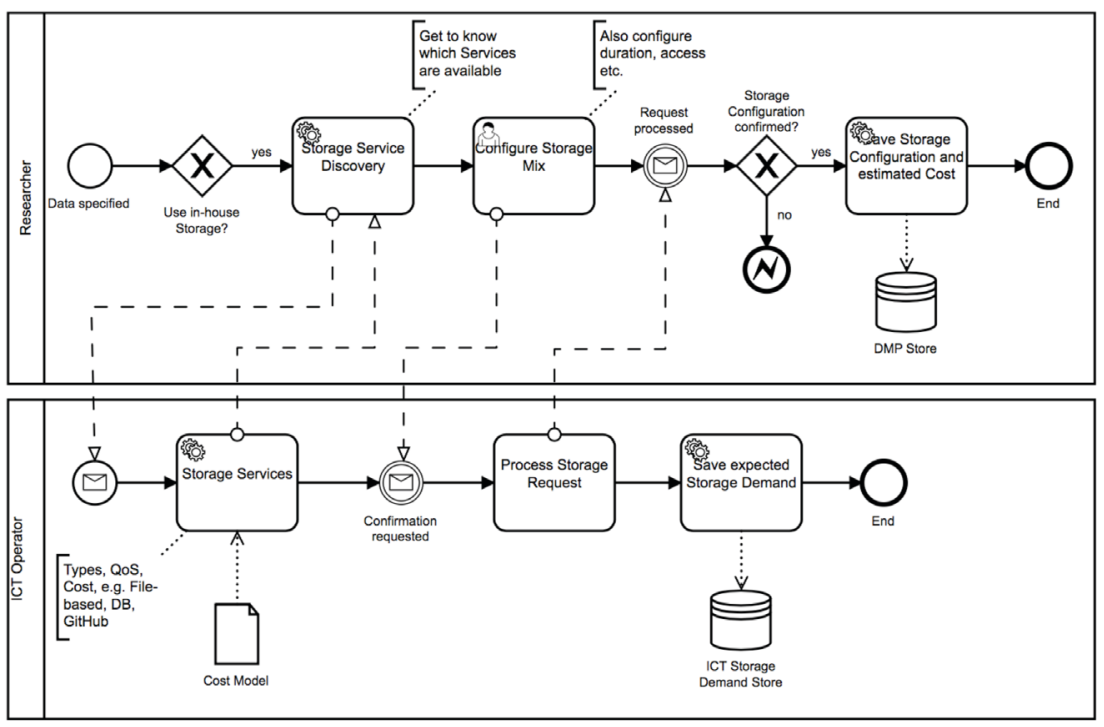

Abb. 3: Beispiel aus DMP Common Standard WG (https://github.com/RDA-DMP-Common/RDA-DMP-Common-Standard)

\subsection{Empfehlungen \& Bedürfnisse}

\section{- Erstellung von DMP-Vorlagen}

Gemeinsam mit Forschenden erarbeitete Vorlagen für (fachspezifische) DMPs vereinfachen die Erstellung der Pläne für die Förderanträge stark. Die Forschenden können sich auf das Einbringen von projektspezifischen Anpassungen konzentrieren und der zusätzliche administrative Aufwand für Forschende wird reduziert. Mittelfristig kann eine zentrale und systematische Ablage der DMPs auch der Planung von organisatorischen und technischen Infrastrukturen dienlich sein.

- Wahl bzw. Implementierung eines DMP-Tools

Die aktuell verfügbaren Online-Werkzeuge zur Erstellung von DMPs sind nur bedingt hilfreich. Mit der Einführung eines lokalen DMP-Tools sollte gewartet werden, bis Anwendungen vorhanden sind, die die wesentlichen Kriterien (u.a. zentrale Steuerung der Rechteverwaltung, Möglichkeit zur Integration in bestehenden Systemen, Versionierung) erfüllen. Die Technische Universität Wien arbeitet derzeit (November 2019) an einem DMP-Tool, welches nach einer individuellen Anpassung in das eigene System integriert werden kann. 


\section{- Aufbau von Beratungen zu DMPs}

Als Ergänzung zu technischen Infrastrukturen wird der Aufbau von institutionellen Strukturen für die Beratung von Forschenden zu DMPs angeregt.

\section{Institutionelle Repositorien ${ }^{27}$}

Forschungseinrichtungen die eScience zukünftig stärker unterstützen wollen, müssen ihre Forschungsinfrastruktur weiter entwickeln. Wesentlicher Bestandteil einer solchen Infrastruktur ist dabei ein institutionelles Repositorium für Forschungsdaten. Forschende können hier ihre Forschungsdaten ablegen und gleichzeitig kann ein solches Repositorium ein Showroom für die Forschung sein und die Vernetzung innerhalb und außerhalb der Forschungseinrichtung fördern. Darüber hinaus bietet das Repositorium die Möglichkeit der Veröffentlichung der Daten der Forschenden wie von den Fördergebern gefordert ${ }^{28}$.

Im Arbeitspaket wurde das beispielhafte Vorgehen zur Auswahl eines institutionellen Repositoriums für Forschungsdaten erarbeitet. Testszenario war das Umfeld der Universität Innsbruck, somit wird im Arbeitspaket die Auswahl eines Long Tail-Repositoriums dargestellt.

Ein institutionelles Repositorium soll möglichst lange einsetzbar sein. Für den Auswahlprozess wurde daher eine Reihe von Anforderungen und Kriterien erarbeitet.

\section{Generelle Anforderungen der Forschenden}

Aus den Interviews mit dem Forschenden aus dem Arbeitspaket Research Lifecycle wurden eine Reihe von Anforderungen extrahiert:

- Teilen von Daten innerhalb und außerhalb der Universität

- Umfassendes System der Rechtevergabe

- Versionskontrolle

- Möglichkeit der Datenrekonstruktion

- Kompatibilität mit der europäischen Datenschutzgrundverordnung

- Nutzbar auch als digitales Archiv

- Einfache Benutzeroberfläche und offene APIs

- Integration von DOIs und personenbezogenen PIDs wie ORCID iDs

- Speicherung sensibler Daten auf universitätseigenen Servern

- OpenAIRE-Kompatibilität

- EU-zertifiziert 


\section{Anwendungsszenarien}

Die Vorgangsweise, Personae zu entwickeln und die Ziele und Bedürfnisse einer Nutzergruppe darzustellen, stammt aus dem Anforderungsmanagement. Eine Persona beschreibt eine Nutzergruppe; ausgehend von dieser Beschreibung werden generelle Ziele und Bedürfnisse erarbeitet, die von der zu evaluierenden Software erfüllt werden müssen. Im Arbeitspaket wurden Personae für eine Reihe von Stakeholdern entwickelt ${ }^{29}$ und daraus die verschiedenen Anwendungsszenarien für das Repositorium erstellt:

- Publikationen

- Supplements

- Zitierfähige Forschungsdaten

- Masterdaten/Rohdaten

- Aktive Forschungsdaten

- Archivierte Forschungsdaten

- Auffinden und Nutzung von Forschungsdaten fremder Forschender

- Showroom der Forschungseinrichtung

\section{Technische Anforderungen}

Die technischen Anforderungen wurden mittels eines Kriterienkatalogs der Research Data Alliance (RDA) geprüft. Die Interessensgruppe für Repository Platforms for Research Data hat eine Matrix mit Anwendungsfällen und funktionellen Anforderungen an Repositorien erstellt ${ }^{30}$. Insgesamt umfasst diese Matrix 39 Kriterien, sie wurde für die kommerziellen Systeme durch die Anbieter und für die Open Source-Systeme durch den Zentralen Informatikdienst ausgefüllt. Unklarheiten bezüglich der Matrix wurden der RDA mit Vorschlägen zu Formulierungen bzw. Beschreibungen zurückgemeldet.

\section{Bedienungsfreundlichkeit}

Die Bedienungsfreundlichkeit wurde anhand praktischer Tests durch Forschende, Bibliotheksangehörige und ZID-Mitarbeiter evaluiert. Die getesteten Systeme wurden anhand von Präsentationen vorgestellt. Anschließend hatten die Teilnehmenden zwei Wochen Zeit zum individuellen Testen. Im Anschluss wurden Kurzfragebögen mit insgesamt zehn Kriterien zur Benutzungsfreundlichkeit der Systeme ausgefüllt ${ }^{31}$. 


\subsection{Ergebnisse}

Für die folgende Systeme wurde die beschriebene Evaluierung durchgeführt und anschließend in Vergleichen untereinander und im Vergleich zwischen Open Source- und kommerziellen Repositorien eine Auswahl getroffen.

\section{Kommerzielle Repositorien}

- Mendeley / Elsevier

- Esploro / ExLibris

- figshare

- TIND.io

\section{Open Source-Repositorien}

- DSpace (Version 6.3)

- Dataverse (Version: v. 4.14)

- invenio (Version v2.0.3)

\section{Open Source-Repositorien vs. kommerzielle Repositorien}

Es gibt deutliche Unterschiede zwischen Open Source und kommerziellen Repositorien. Generell sind beide Lösungen für ein institutionelles Repositorium möglich. Eine allgemeine Empfehlung für ein bestimmtes System wird hier nicht gegeben, da jedes System seine eigenen Chancen und Risiken birgt und die Lösung in die jeweilige Infrastrukturumgebung der Universität eingebettet werden muss. Falls die Präferenz für eine Open Source oder für eine kommerzielle Lösung frühzeitig geklärt wird, kann auf den Test anderer Lösungen gegebenenfalls verzichtet werden (z.B. Usability). Die Präferenz kann unter anderem von diversen Faktoren abhängen:

- Generelle Präferenz für Open Source- oder kommerzielle Lösungen innerhalb der Forschungseinrichtung

- Kostenvergleich von Preisen kommerzieller Produkte vs. Arbeitskraft und weitere Ressourcen (z. B. Speicher)

- Weitere mögliche Einflussfaktoren: Flexibilität, Stabilität, Quality of Service (24/7), Zukunftssicherheit, Möglichkeit zur Einbindung bestehender interner Systeme 


\section{Open Source-Repositorien}

Sie sind schnell installiert und bieten größtmögliche Freiheit bei der Erfüllung der Anforderungen der Anwender, aber sind aufwendiger für den Betrieb durch den Zentralen Informatikdienst. Für Aufbau, Betrieb und Weiterentwicklung von Open Source-Repositorien sind entsprechende Kostenschätzungen durchzuführen. Getestet wurden die Open SourceSysteme DSpace, Dataverse und invenio.

Folgendes ist bei der Nutzung von Open Source-Repositorien zu beachten:

- Personal- und Speicherkosten: Für den Betrieb eines lokalen Systems muss eine Person verfügbar sein und die Speicherkosten müssen passend zu den Richtlinien zur Speicherung und zum Teilen von Daten geschätzt werden.

- Support: Es werden zeitgemäße Betriebsstandards benötigt, die an die aktuellen Lebens- und Arbeitsumstände der Forschenden angepasst sind.

- Kontinuierliche Weiterentwicklung: Das Repositorium muss laufend upgedatet werden, zusätzliche oder neue Infrastruktur zur Unterstützung oder Ergänzung des Angebots muss aufgebaut werden.

- Zeitlicher Ablauf: Ein angemessener Zeitplan für die Installation ist beim Zeitplan zu berücksichtigen.

- CoreTrustSeal-Zertifizierung: In drei bis fünf Jahren wird von Seiten der Fördergeber die Datenablage in ein zertifiziertes Repositorium gefordert werden. Die Anforderungen ${ }^{32}$ betreffen u.a. eine maximale Serviceverfügbarkeit, eine Spiegelung der Daten in mehreren hundert Kilometern Entfernung, Bandbreite, die für die globalen und lokalen Anforderungen ausreicht.

Die Open Source-Systeme wurden ähnlich den kommerziellen Repositories bewertet. Auch bei diesen Systemen wurden die Kriterien der RDA-Matrix herangezogen. Lediglich der Test der Usability wurde verkürzt anhand von Demo-Systemen im Internet durchgeführt.

\section{Kommerzielle Produkte}

Sie garantieren Quality of Service, aber bei Änderungen der Dienste und bei der Entfernung der Daten ist man auf die Anbieter angewiesen. Es wurden zwei Arten von kommerziellen Repositorien getestet. Zum einen sogenannte Online-Dienste, bei denen eine Softwarelösung für viele Kunden zentral 
betrieben wird (im Test: Esploro / ExLibris, Mendeley / Elsevier und figshare), und zum anderen eine Software as a Service Lösung (im Test: TIND.io), bei der der Anbieter eine eigene Instanz der Anwendung für den Kunden betreibt. Die Online-Dienste bieten als Einstiegspunkt für den Kunden eine eigene Startseite in ihrer Plattform an, die optisch angepasst werden kann. Das Onboarding bei einer solchen Lösung geht dementsprechend schnell und ist kostengünstig. Bei der Software as a Service Lösung hat der Kunde nicht nur größere Gestaltungsmöglichkeiten, was das Erscheinungsbild betrifft, er kann auch Einfluss auf die verwendete Infrastruktur nehmen und sich zusätzliche Funktionalitäten einbauen lassen. Das Onboarding ist bei einer solchen Lösung teurer und dauert länger.

\subsection{Vergleich der Open Source-Repositorien}

invenio $^{33}$

invenio wurde von CERN entwickelt und verwaltet dort Millionen Dokumente und Daten. invenio ist den meisten Forschenden bekannt, da es die Software hinter zenodo.org ist, dem Repositorium der EU.

Vorteile:

- einfache Bedienung

- hoher Funktionsumfang

- Communities zum Erstellen abgeschlossener Bereiche

- OpenAIRE compliant

- EU endorsed

- Unterstützung des SWORD-Protokolls

- modulbasiert, einfach erweiterbar

- moderne Applikationsarchitektur mit WSGI Frameworks, Angular JS basierter Oberfläche, Python

- erfüllt alle Kriterien der RDA-Matrix

\section{Dataverse $^{34}$}

Dataverse wurde ursprünglich von der Universität Harvard entwickelt und dann als Open Source-Produkt freigegeben.

Vorteile:

- $\quad$ einfache Bedienung

- $\quad$ hoher Funktionsumfang 
- $\quad$ eigene Dataverses für spezielle Anforderungen

- $\quad$ Unterstützung des SWORD-Protokolls

- $\quad$ erfüllt alle Kriterien der RDA Matrix

Nachteile:

- nicht durchgängig modulare Systemarchitektur

DSpace $^{35}$

DSpace wurde ursprünglich als Repositorium für Publikationen entwickelt und um die Fähigkeit zur Verwaltung von Forschungsdaten erweitert. Derzeit wird DSpace um eine völlig neue Oberfläche erweitert, die im Jahr 2019, folglich erst nach den Tests fertig gestellt wurde.

Vorteile:

- große Nutzer- und Entwickler-Community

- Unterstützung des SWORD-Protokolls

Nachteile:

- eingeschränkte Integrity-Checks

- keine Versionierung von Daten

- keine Erfüllung des OASIS-Standards ${ }^{36}$-> Somit erfüllt die getestete DSpace-Version wesentliche Kriterien der RDA-Matrix nicht

\subsection{Vergleich der kommerziellen Repositorien}

TIND.io ${ }^{37}$

TIND.io ist eine Ausgründung von CERN und verwendet invenio als Basis für seine Produkte. TIND.io ist das einzige Software as a Service Repositorium im Vergleich.

Vorteile:

- Software as a Service: Repositorium „gehört" der Universität

- Einfache Exit-Strategie: Eigene Server können genutzt bzW. AWS-Infrastruktur kann übernommen werden, die TIND.io Plattform kann durch invenio-Software ersetzt werden

- Dreifache Sicherung der Daten

- Erfüllung aller Kriterien der RDA-Matrix Sehr positive Beurteilung der Usability 
Nachteile:

- Längerer Onboarding Prozess als bei Onlinediensten

- Die technischen Voraussetzungen für die Zertifizierung nach CoreTrustSeal sind bereits gegeben, die Zertifizierung muss aber von jeder Universität selbst durchgeführt werden

figshare $^{38}$

Laut Angaben von figshare bietet das All-in-One-Repositorium „a home for papers, FAIR data and non-traditional research outputs that is easy to use and ready now ${ }^{39}$ ".

Vorteile:

- Daten in lokalen Speichern können im Repositorium verortet werden

- Mögliche Exit-Strategie durch Unterstützung des SWORD-Protokolls ${ }^{40}$

- Erfüllung aller Kriterien der RDA-Matrix

- Positive Beurteilung der Usability

\section{Mendeley / Elsevier ${ }^{41}$}

Mendeley ist laut Elsevier ein Reference Manager und ein akademisches soziales Netzwerk, in dem Forschende ihre wissenschaftliche Arbeit organisieren, mit anderen online zusammenarbeiten und aktuelle Forschungsergebnisse finden können ${ }^{42}$.

Vorteile:

- Einfach zu bedienende Oberfläche

- Erfüllung aller Kriterien der RDA-Matrix

\section{Esploro / ExLibris ${ }^{43}$}

Nach Angaben von ExLibris bietet die cloudgestützte Forschungsdienstplattform Esploro die systematische Erfassung, Verwaltung und Verbreitung von Forschungsergebnissen und -daten, die Nutzung der Bibliothekskompetenz und -technologie sowie die nahtlose Integration in bestehende Forschungsabläufe $e^{44}$.

Vorteile:

- persönliche Website für Forschende, die automatisch mit Daten aus dem Netz angereichert wird 
Nachteile:

- Erfüllt derzeit nicht alle Kriterien der RDA-Matrix

\subsection{Empfehlungen \& Bedürfnisse}

- Einrichtung eines institutionellen Repositoriums, Zugang zu einem Repositorium

Jede Institution wird für die Veröffentlichung der eigenen Forschungsdaten ein Ablagesystem für Daten benötigen, welche in internationalen oder nationalen fachspezifischen Repositorien nicht abgelegt werden können. Ein solches Repository wird als Long Tail-Repository bezeichnet, der benötigte Umfang ist abhängig von der Forschung an der jeweiligen Institution. Möglich sind auch gemeinsam betriebene und/oder genutzte Repositorien von mehreren Forschungseinrichtungen.

- Klassifikation von Forschungsdaten Unabhängig von der Wahl eines Repositorys sind Richtlinien für die Ablage und Speicherung von Forschungsdaten für die Forschenden notwendig. Verschiedene Arten und Versionen von Daten (Rohdaten, Masterdaten, sensible Daten, zitierfähige Daten, usw.) sollten in dafür vorgesehenen Systemen gemäß der FAIR-Prinzipien abgelegt, geteilt und/oder veröffentlicht werden.

- Entscheidung zur Offenheit eines Repositoriums

Vor der Implementierung eines Repositoriums kann das Open Access Spectrum (OAS), welches verschiedene Themen wie Zugriffs-, Verwertungs-, Urheber- und Veröffentlichungsrechte sowie automatische Veröffentlichung, Maschinenlesbarkeit umfasst, zur Definition der Offenheit dienen. Im Allgemeinen wird empfohlen, das Repositorium so offen wie möglich, so geschlossen wie notwendig zu gestalten. Darüber hinaus sind individuelle Einstellungen bezüglich der Offenheit der eigenen Daten seitens der Forschenden möglich.

- Systemdesign für ein nachhaltiges Repositorium mit Möglichkeit zur Zertifizierung

Zur Sicherstellung der Nachhaltigkeit bzw. der Zertifizierung eines Repositoriums sind die Kriterien von re3data.org bzw. die Anforderungen für eine Zertifikation (z.B. CoreTrustSeal) bereits bei der Implementierung eines Repositoriums zu berücksichtigen. Eine Zertifizierung dient neben dem Nachkommen der Empfehlung seitens der Fördergeber insbesondere der Klärung interner Prozesse in der Forschungseinrichtung. 


\section{- ORCID iDs im Repositorium}

Bevor mit der Implementierung des gewählten Repositoriums begonnen werden kann, ist der Umgang mit ORCIDs im Repositorium zu klären. Alle im Projekt getesteten Systeme verfügen über Module zum Authentifizieren mittels ORCID iD und weisen diese anschließend den gespeicherten Daten zu. Verwendet eine Institution diese persistenten Identifikatoren bei mehr als einem System zum Authentifizieren wird eine Mitgliedschaft im ORCID-Konsortium benötigt.

- Vermittlung von Kompetenzen bezüglich Lizenzen und Copyrights Die Angaben von Lizenzen und Urheberrechten werden zur Veröffentlichung von Forschungsdaten in Repositorien unumgänglich sein. Forschende äußerten den Wunsch nach Beratung zu diesem Thema.

- Steigerung der Sichtbarkeit des eigenen Repositoriums Eine Multikanalverbreitung macht das eigene Repositorium sichtbarer und kann Mehrwerte für (potentielle) User aufzeigen (z.B. die Sicherung von Roh- und Masterdaten). In diesem Prozess können auch Informationen zu den Verantwortlichkeiten seitens User und Repositorienbetrieb bereitgestellt werden.

\section{Metadaten ${ }^{45}$}

Im Vorgängerprojekt e-Infrastructures Austria wurde bereits intensiv über unterschiedliche Aspekte von Metadaten gearbeitet und mehrere Vorarbeiten geleistet. Diese Arbeiten wurden zum Teil weitergeführt und mit neuen Aspekten ergänzt, wobei in diesem Projekt versucht wurde, das Thema „Metadaten“ aus zusätzlichen Blickwinkeln zu sehen. Metadaten sind mehr als nur Daten über Daten, sie können in der Forschung unterschiedliche Rollen haben. Zum Teil dienen sie der Zugänglichkeit zu den Daten, wie beispielsweise zu Messdaten, sie können aber auch selbst zum Gegenstand von Forschung werden, wie beispielsweise in der Literaturwissenschaft. Metadaten lassen sich aber auch aus technischer Sicht betrachten, sie sind Gegenstand der Überlegung, wie man sie möglichst automatisch generieren kann um den Forschenden Arbeit zu ersparen. Für Bibliotheken ist der Umgang mit Metadaten seit jeher selbstverständlich, Metadaten in Repositorien stellen jedoch eine neue Herausforderung dar, das Wissen darüber, wie man sie generiert, pflegt, zur Verfügung stellt, mit anderen teilt und langfristig archiviert, muss erst nach und nach erarbeitet werden, am besten zusammen mit Experten aus der IT. 


\subsection{Umgang mit Metadaten in Repositorien, eine Österreichweite Umfrage. Zweite Folge}

Der Umgang mit Metadaten in unterschiedlichen Repositorien hat sich in den letzten Jahren geändert. Die Umfrage aus dem Vorgängerprojekt „Umgang mit Metadaten in Repositorien an österreichischen Universitäten “46, sollte im Nachfolgeprojekt erneut durchgeführt werden. Zwischen den beiden Umfragen liegen ca. drei Jahre, in denen sich die Repositorienlandschaft stark verändert hat. Es wurden neue Repositorien in Betrieb genommen und Services dazu aufgebaut. Es war deshalb anzunehmen, dass sich in der Zwischenzeit auch der Umgang mit Metadaten verändert hat. Die vermutete Entwicklung sollte dokumentiert und Fortschritte im Vergleich der beiden Fragebogenpakete herausgearbeitet werden. Um dies zu ermöglichen, wurde entschieden, auch jene Institutionen zur Befragung einzuladen, die zwar beim ersten Projekt e-Infrastructures Austria, aber nicht mehr bei e-Infrastructures Austria Plus dabei waren. Um zu vergleichbaren Ergebnissen zu gelangen, wurde der Fragebogen nicht komplett neu gestaltet, sondern nur leicht überarbeitet. Einige Fragen wurden basierend auf den Erfahrungen des Jahres 2015 ergänzt bzw. weggelassen. Auch die Form des Telefoninterviews und die sonstige Vorgangsweise wurden beibehalten, insbesondere die Möglichkeit, den Fragebogen schon vorab unter Hinzuziehung von Experten an der eigenen Institution auszufüllen und eventuelle Unklarheiten im Zuge des persönlichen Gesprächs zu klären. Den Interviewpartnern wurde auch diesmal wieder Anonymität zugesichert, d.h. die Rohdaten aus den einzelnen Fragebögen werden archiviert, aber nicht ungefiltert an die Öffentlichkeit weitergegeben. Der adaptierte Fragebogen wurde Anfang August 2018 verschickt, die Interviews wurden im Zeitraum September 2018 bis Dezember 2018 geführt. Ein wesentliches Ergebnis ist, dass alle befragten Einrichtungen mittlerweile zumindest über ein Repositorium für Publikationen verfügen, was 2015 noch nicht der Fall war. Außerdem ist zu erkennen, dass der Bereich der Forschungsdaten mehr Aufmerksamkeit erhält als noch vor vier Jahren. Die Archivierung von Publikationen und auch von Forschungsdaten wurde wichtiger. Auch eine mögliche Nachnutzung der Daten wurde stärker in den Fokus gerückt. Ein Trend scheint auch die fortschreitende Automatisierung von Abläufen zu sein, auch werden mehr Templates und Ausfüllhilfen angeboten. Die inhaltliche Kontrolle der archivierten Daten hat dagegen abgenommen. Auffallend ist auch, dass weniger Daten ohne Metadaten als 2015 archiviert werden, auch hier könnte man von einer Professionalisierung sprechen. Leider gilt das nicht für die Barrierefreiheit, hier ist kein nennenswerter 
Fortschritt zu erkennen. Wie die Zukunft von Repositorien, vom Umgang mit Daten und Metadaten, aussehen wird, lässt sich schwer abschätzen. Wichtig scheint jedoch zu sein, so früh und so eng wie möglich mit den Forschenden zusammenzuarbeiten, um genau dort Unterstützung geben zu können, wo sie gebraucht wird, möglichst rasch und unkompliziert. Gleichzeitig sollten auch die technischen Trends im Auge behalten werden, die den Umgang mit Metadaten eventuell erleichtern können. Dabei ist eine gewisse Offenheit und Flexibilität nötig. Wo beispielsweise eine Öffnung der Daten oder auch der Metadaten aus ethischen oder rechtlichen Gründen nicht möglich ist, wird es neue Lösungen geben müssen. Das Thema Metadaten wird jedoch sicher nicht an Wichtigkeit verlieren, denn nur so ist die Auffındbarkeit der Daten, deren Menge weiterhin rasant zunehmen wird, auch in Zukunft gegeben.

\subsection{Zum Umgang mit Metadaten an der ÖAW: Eine erste Annäherung ${ }^{47}$}

An der Österreichischen Akademie der Wissenschaften (ÖAW) werden seit einigen Jahren verschiedene Digitalisierungsprojekte durchgeführt, deren Ergebnisse online zugänglich gemacht werden. Den Forschenden stehen mit A Research Centre for the HumanitiEs (ARCHE) und epub.oeaw zwei große Repositorien zur Verfügung. Wie bekannt sind vorhandene Infrastrukturen und welche Anforderungen haben die Forschenden an diese Systeme? Wie gehen die Forschenden mit Metadaten um? Um einen Einblick in diese Fragen zu bekommen, wurde im Frühjahr 2019 an der ÖAW eine vorerst kleine Umfrage unter Personen aus verschiedenen Disziplinen der Geisteswissenschaften (sowie der Bibliothek) gestartet. Die Umfrage war auf einem Teil der Umfrage aus dem Vorgängerprojekt e-Infrastructures Austria ${ }^{48}$ aufgebaut und wurde schriftlich durchgeführt. Diese Umfrage ist als erster Schritt in einer umfassenderen Auseinandersetzung mit dem Thema zu sehen, da Metadaten für die Auffindbarkeit, die Nachnutzung und die Archivierung von digitalen Daten unentbehrlich sind.

\subsection{Metadaten für Forschungsdaten: Bedürfnisse und Anforderungen in den Naturwissenschaften ${ }^{49}$}

Bei der Entwicklung von bedarfsgerechten Services im Bereich eScience sind Untersuchungen der Bedürfnisse und Anforderungen der Zielgruppen von besonderer Relevanz. Angelehnt an die Grounded Theory wurden von April bis Mai 2019 acht Leitfadeninterviews mit Naturwissenschaftlern der Universität Wien und Medizinern der Medizinischen Universität Wien 
durchgeführt. Die Ergebnisse zeigen, dass ein Verständnis des Begriffes „Metadaten" unter Forschenden noch nicht flächendeckend vorhanden ist. Es konnten aber mehrere Beispiele der Beschreibung von Daten identifiziert, sowie sechs Bereiche an Wünschen der Forschenden ausgearbeitet werden. Diese beschäftigen sich u.a. mit der Notwendigkeit, Services zweisprachig zu konzipieren, mit den Anforderungen an technische Infrastruktur oder dem Wunsch nach dem Einsatz von Data Stewards, die Fachkenntnisse aus einer relevanten Disziplin mitbringen.

\subsection{Metadatenmapping. Die Gegenüberstellung verschiedener Metadaten- schemata am Beispiel UWMETADATA $\gg$ MODS $3.6^{50}$}

Am Beispiel von Phaidra (Repositorium der Universität Wien) soll die Vorgehensweise bei einem Metadatenmapping aufgezeigt und analysiert werden. Das Mapping wurde in Zusammenarbeit mit der Universität Padua im Frühjahr 2018 erstellt.

\subsection{Automatische Übertragung von Metadaten in Videodateien ${ }^{51}$}

Unter dem Begriff „automatische Übernahme von Metadaten“ soll in diesem Dokument das programmgesteuerte Auslesen und Speichern von Tags aus Videodateien verstanden werden. Untersucht wurden dabei die beiden wichtigsten Video-Containerformate Matroska (MKV) und MP4. In der Publikation wird das Anreichern von Videodateien um Metadaten, als auch das Auslesen vorhandener Metadaten beschreiben. Beide Vorgänge werden zu verschiedenen Zwecken eingesetzt. Das Anreichern der Videodateien wird benötigt, um projektspezifische Informationen in die Metadaten der Videodateien einzubringen, das Auslesen aus den Videodateien wird verwendet um Metadaten automatisch zu extrahieren wenn eine Datei in ein Repositorium eingebracht wird.

Für beide Formate wurde eine Reihe von Werkzeugen und Anwendungsfälle getestet und letztlich eine Empfehlung für das Matroska-Format abgegeben, die ausführlich im Endbericht nachzulesen ist.

\subsection{Sacherschliessung von Forschungsdaten ${ }^{52}$}

Der Beitrag stellt die Frage nach der inhaltlichen Erschließung von Forschungsdaten als Teil ihrer Metadaten. Ein Forschungsprojekt das Daten produziert kann nicht voraussehen in welcher Weise die entstehenden Daten nachgenutzt werden. Kann eine Sacherschließung im bibliotheka- 
rischen Sinne die Auffindbarkeit der Daten für eine Nachnutzung gewährleisten?

Um einen Eindruck zu erhalten, wie Institutionen die Repositorien für Forschungsdaten unterhalten mit diesem Thema umgehen, wurden die Forschungsdaten-Services von Institutionen in Deutschland, Großbritannien, Holland, der Schweiz und Frankreich kontaktiert. Von den neun kontaktierten Institutionen haben sieben die per E-Mail gestellten Fragen beantwortet. Die Antworten zeigen drei Herangehensweisen an die Inhaltliche Erschließung von Forschungsdaten:

- Die Forschungsdaten von den Forschenden mit selbst gewählten Stichworten beschlagwortet.

- Dazu kann eine klassifikatorische Erschließung (z.B. Dewey Decimal Classification) kommen.

- Einige Institutionen sehen einen Mehrwert in einer aufwändigen, weil durch Fachkräfte zu leistenden, vertieften Erschließung, andere nicht.

In einem weiteren Schritt wurden online Datenbanken für Wissensorganisationssysteme, Standards und Policies auf ihre Eignung untersucht, den Forschenden und Repository-Managern fachspezifische kontrollierte Vokabulare vorzuschlagen. Von sechs untersuchten Datenbanken können nur BARTOC $^{53}$ und FAIRsharing ${ }^{54}$ empfohlen werden.

\subsection{Interviews zu Forschungsdaten ${ }^{55}$}

An der Universität Wien wurden Forschende unterschiedlicher Disziplinen zu ihren Forschungsdaten und dem Lifecycle ihrer Daten interviewt. Die Interviews sind in Phaidra publiziert und wurden im September 2019 frei geschalten.

\subsection{Austausch mit dem HRSM-Projekt „Portfolio/Showroom - Making Art Research Accessible 6 (656}

Das Forschungsinformationssystem (CRIS) „Portfolio/Showroom“, das auf Open Source-Basis aktuell an der Universität für angewandte Kunst Wien (Angewandte) gemeinsam mit den Projektpartnern entwickelt wird ${ }^{57}$, wählte mit dem Ontology-Driven Software Development sowie dem UserDriven Development einen neuen Ansatz in der Entwicklung von Software. Usability und User Experience (UX) stehen im Mittelpunkt der Konzeption des CRIS. 


\subsection{Ausblick}

Die Ergebnisse der Unterarbeitsgruppen zeigen, wie unterschiedlich an das Thema "Metadaten“ in Repositorien herangegangen werden kann. Die Beschreibungen der Daten werden umso wichtiger, je automatisierter die Datenabfrage gestaltet ist - Linked Data, die Verwendung von Thesauri und genormten Daten werden für die Auffindbarkeit der Daten immer wichtiger.

\subsection{Empfehlungen \& Bedürfnisse}

- Automatisiertes Einfügen allgemeingültiger Metadaten

Unabhängig vom Fachbereich sind allenfalls Ort, Zeit und die Projektnummer der Fördergeber möglichst automatisiert in den Metadaten anzugeben (z.B. Austrian Science Fund (FWF): project number). Für bestimmte Dateiformate sollen Möglichkeiten eruiert werden, wie auch inhaltliche Metadaten direkt aus den Dateien ausgelesen werden können.

- Vermittlung von Kompetenzen zum Umgang mit Metadaten

Kenntnisse zum Umgang mit Metadaten seitens der Forschenden werden benötigt, um die eigenen Forschungsdaten im Sinne der FAIR Prinzipien mit den entsprechenden Metadaten auffindbar und nachnutzbar für andere Forschenden zu machen.

- Barrierefreie Metadaten

Metadaten sind möglichst barrierefrei für alle User zu gestalten. Dazu zählen unter anderem die Mehrsprachigkeit und die Verständlichkeit auch für Personen aus anderen Fachdisziplinen. In den Metadaten sollte außerdem ein Hinweis auf barrierefreie Inhalte enthalten sein.

- Disziplinnahe Unterstützung bei der Metadatenvergabe Wichtig ist der Aufbau von Strukturen zur Unterstützung der Forschenden bezüglich Metadaten, zum Beispiel durch die einzelnen Fachbereiche. Ergebnisse aus der Gruppe „Research Data Alliance“ und der Initiative "GoFAIR" zu fachspezifischen Metadatenstandards sind hier miteinzubeziehen.

- Kontrollierte Vokabularien

Zur Erfüllung der FAIR-Prinzipien wird die Nutzung von kontrollierten Vokabularien oder Thesauri zur Klassifizierung der Daten nach offiziellen Schemata und zur Kennzeichnung des Inhalts mit einheitlichen Schlüsselwörtern empfohlen (wie zum Beispiel ÖFOS 2010). 


\section{Persistente Identifikatoren ${ }^{58}$}

Das Arbeitspaket beschäftigte sich mit den persistenten Identifikatoren DOI (Digital Object Identifier) und ORCID iD (Open Researcher and Contributor ID) in Bezug auf organisatorische Rahmenbedingungen an österreichischen Institutionen sowie auf deren praktische Verwendung.

\subsection{DOI}

„Ein Digital Object Identifier (DOI; deutsch Digitaler Objektbezeichner) nach ISO 26324 soll ein eindeutiger und dauerhafter digitaler Identifikator für physische, digitale oder abstrakte Objekte sein. [...] Verantwortlich für Integrität und Dauerhaftigkeit eines DOI ist die Organisation, die auch die Verantwortung für das jeweilige Objekt trägt. Das DOI-System baut auf dem Handle-System auf und ist vereinfacht ausgedrückt mit ISBN und ISSN vergleichbar, soll jedoch durch eine integrierte Lokalisierungsfunktion darüber hinausgehen ${ }^{59}$."

DOls sind in den letzten Jahren zum De-facto-Standard als eindeutige, dauerhafte, zitierbare Identifikatoren für Publikationen und Forschungsdaten geworden. In Österreich gab es jedoch zu Beginn des Projekts keine zentrale Registrierungsstelle, die die DOI-Vergabe administriert; an einzelnen Institutionen waren bereits Individuallösungen im Einsatz.

Die Initiative des Arbeitspakets im Bereich DOI, die mit der Erstellung eines Entscheidungspapiers zum Thema „National DOI Desk“ für den Lenkungsausschuss des Projekts begann, konnte zu einem erfolgreichen Abschluss gebracht werden: Die Technische Universität Wien erklärte sich nach mehreren Gesprächen im Rahmen des Arbeitspakets bereit, die DOIVergabe für österreichische Institutionen von der TIB Hannover zu übernehmen. Eine Servicestelle für die DOI-Vergabe in Österreich ist derzeit an der Technischen Universität Wien im Aufbau. Damit steht in Österreich erstmals ein lokaler Ansprechpartner zum Wissenstransfer und zur Koordination im Bereich der DOI-Vergabe zur Verfügung. Auf der Basis vorhandener Leitfäden und Policies erstellten die Mitglieder des Arbeitspakets außerdem eine Muster-Policy für die DOI-Vergabe an (österreichischen) Institutionen. Darin werden Rechte und Pflichten von DOI-BezieherInnen und Anforderungen an digitale Objekte definiert sowie Zuständigkeiten und Workflows für die Vergabe von DOIs festgelegt. Die Muster-Policy findet sich im Anhang des Endberichts und wurde im April 2019 in einer deutschen und einer englischen Fassung veröffentlicht und zur Nachnutzung bereitgestellt. 


\subsection{ORCID}

„ORCID provides a persistent digital identifier that distinguishes you from every other researcher and, through integration in key research workflows such as manuscript and grant submission, supports automated linkages between you and your professional activities ensuring that your work is recognized ${ }^{60}$."

ORCID iDs ermöglichen eine eindeutige Zuordnung von Forschungsleistungen zu Personen, weltweit wurden bereits über 6 Millionen ORCID iDs vergeben. Zur Information über diese persistenten Identifikatoren für Forschende und zur Interessenerhebung für ein nationales Konsortium veranstaltete das Arbeitspaket im Mai 2018 gemeinsam mit der Universität für Bodenkultur Wien den ORCID Austria Workshop ${ }^{61}$ und führte eine mehrstufige Umfrage zu möglichen Interessenten an einem solchen Konsortium und zum Consortium Lead durch. Auf Basis dieser Vorarbeiten haben sich die Technische Universität Wien und die Universität Wien geeinigt, ab 1.1.2019 gemeinsam den Lead eines ORCID Austria Consortiums zu übernehmen, an dem derzeit 11 österreichische Institutionen teilnehmen. Auf der Website des Konsortiums ${ }^{62}$ sind weitere Informationen zu finden, im Endbericht des Projekts findet sich außerdem ein Exkurs über die Möglichkeiten ORCIDs mittels APIs stärker in der Institution zu verankern.

\subsection{Empfehlungen \& Bedürfnisse}

- PIDs für publizierte Forschungsdaten

Für einen permanenten Zugang zu publizierten Forschungsdaten wird die Verwendung von etablierten PIDs empfohlen. Zum jetzigen Zeitpunkt sind insbesondere DOls und ORCID iDs relevant. Beim Aufbau neuer eScience-Infrastruktur sollte daher bereits bei der Spezifizierung darauf geachtet werden, dass die entsprechenden Schnittstellen der PID-Registrierungsagenturen unterstützt werden, um Forschungsleistungen möglichst automatisiert mit PIDs zu versehen.

- Implementierung einer institutionellen DOI-Policy

Die Regeln für die Vergabe von DOIs an der eigenen Institution sollte formalisiert werden, idealerweise über eine institutionelle DOI-Policy. Als Basis dafür kann die DOI-Muster-Policy aus dem vorliegenden Projekt verwendet werden.

- Erstellung eines lokalen PID-Graphs für die eigene Institution Das EOSC Projekt FREYA beschäftigt sich mit Infrastrukturen zu per- 
sistenten Identifikatoren und schlägt die Erstellung eines sogenannten PID-Graphen vor. Ein PID-Graph, der die Beziehungen der Daten durch verwendete PIDs visualisiert, hilft bei der Dokumentation und Weiterentwicklung der Implementierung und Nutzung von PIDs in der Infrastruktur der eigenen Institution.

- Überlegungen zum Umgang mit nicht-publizierten Forschungsdaten im Zusammenhang mit PIDs

Klärungsbedarf besteht darin, wie bei der Vergabe von PIDs mit nichtpublizierten Forschungsdaten verfahren werden soll. Ein Minimumkriterium der meisten PID-Registrierungsagenturen ist nämlich, dass zumindest die zugehörigen Metadaten öffentlich sind.

\section{GO FAIR ${ }^{63}$}

Das Arbeitspaket hatte sich zum Ziel gesetzt, vorhandene und publizierte Dokumente im österreichischen Netzwerk zu teilen und zu diskutieren sowie persönliche Kontakte mit bereits stattfindenden Initiativen im europäischen Ausland zu knüpfen und diese zu pflegen.

\subsection{Workshop „Linking Open Science in Austria“}

An dieser zweitägigen Veranstaltung, die gemeinschaftlich vom OpenAIRE NOAD Austria Team und dem Verbindungsbüro für e-Infrastruktur - beide an der Universitätsbibliothek Wien beheimatet - organisiert wurde, trafen sich rund 60 nationale und internationale Teilnehmende und Experten mit unterschiedlichem fachlichem Hintergrund: von Bibliotheksmitarbeitern über Repository Managern, Mitarbeiter der Forschungsservices bis hin zu Forschenden sowie zu politischen Entscheidungsträgern und Fördergebern. Die European Open Science Cloud (EOSC) ist eine der aktuellen Triebkräfte für die Erschließung von Forschungsdaten. Die Bedeutung lokaler Veranstaltungen mit internationalem Publikum wurde durch die Anwesenheit von Mitgliedern des EOSC-Exekutivrats, des EOSC-Verwaltungsrats und des EOSC-Sekretariats sowie mehrerer EOSCUnterstützungsprojekte unterstrichen. Durch diese Veranstaltung war es möglich, sich mit dem Status Quo bestehender Service-Infrastrukturen, die bereits die FAIR-Prinzipien anwenden, vertraut zu machen. Ein roter Faden während der gesamten Veranstaltung war die Frage, was erforderlich ist, um die Umsetzung von FAIR auf nationaler und internationaler Ebene voranzutreiben, die Schaffung universeller Standards zu be- 
schleunigen, FAIR-Daten zu unterstützen und FAIR-Forschungsergebnisse zu erstellen. Zur Unterstützung von FAIR-Daten und FAIR-Diensten als wesentlicher Bestandteil der Umsetzung von EOSC wird empfohlen, solche Veranstaltung in anderen Ländern und Institutionen zu wiederholen. Die Veranstaltung zeigt eine Best Practice, wie Bedürfnisse und Wünsche gesammelt und wie Hindernisse überwunden werden können, um eine Kultur offener Forschungsdaten in der Gemeinschaft von Research Facilitators, Bibliotheksmitarbeitern, politischen Entscheidungsträgern und Forschenden zu stärken.

- Webpage des Events „Linking Open Science in Austria“. https://linkingopenscience.univie.ac.at/

- OpenAIRE-Blog. https://www.openaire.eu/blogs/connect-andget-connected-linking-openscience-in-austria

\subsection{Certification Workshop on FAIR-aligned repositories in Austria}

Zudem war ein „Certification Workshop on FAIR-aligned repositories in Austria" zum Thema FAIR und Zertifizierung mit dem Data Archiving and Networked Services (DANS) ${ }^{64}$, Niederlande für den 14. November 2019 angesetzt ${ }^{65}$. Ziel ist die geographische Abdeckung der österreichischen Forschungslandschaft als EOSC-vorbereitende Maßnahme und zur Vorbereitung von FAIR Alignment für Daten und Services in Österreich. Es werden Einblicke in die CoreTrustSeal-Zertifizierung gegeben, in den Erwerb und in die Initiierung eines Netzwerks von Experten, die sich auf die Zertifizierung vorbereiten können. Die Zielgruppe besteht aus Repository Manager aus möglichst vielen österreichischen Forschungseinrichtungen, die derzeit Forschungsdatenmanagement betreiben. Die Teilnahme ist nicht an der Beteiligung am Projekt e-Infrastructures Austria Plus gebunden.

\subsection{Empfehlungen \& Bedürfnisse}

- FAIR Data and Infrastructure Certification In der „EOSC Fair Working Group“ und im „FAIRsFAIR“ Projekt der EU wird eine „FAIR Data Certification” von Infrastruktur gefordert. Damit soll die Interoperabilität von eScience Infrastrukturen auch zwischen verschiedenen Fachbereichen erhöht werden. Ein „FAIR Data Certification Scheme" ist in Ausarbeitung und sollte bei der Implementierung lokaler eScience Infrastruktur beachtet werden. 


\section{Zusammenfassung des Projektmanagements}

2016 war durch das Vorgängerprojekt e-Infrastructures die Etablierung geeigneter Plattformen gelungen, die den Austausch zwischen den Partnerinstitutionen ermöglichten und Bibliotheken, IT-Bereiche, Forschungsservices und Forschende vernetzten. Diese Verbindungen bildeten die Grundlage für das aktuelle Projekt e-Infrastructures Austria Plus. Mit der Medizinischen Universität Innsbruck und der UMIT Hall in Tirol konnten zwei neue Partnerinnen für das Projekt gewonnen werden. Das Projekt hatte mit neun Projektpartnern deutlich weniger teilnehmende Institutionen als das Vorgängerprojekt und mit der Fördersumme von EUR 300.000,- ein äußerst begrenztes Budget. Aus den Anforderungen der Projektpartner und unter Einbeziehung der Ergebnisse des Vorgängerprojekts wurden im Projekt sieben Arbeitspakete aus unterschiedlichen Bereichen des Forschungsdatenmanagement definiert. Von FDM-Policies, über die Abbildung von Forschungsprozessen, der Erfassung von Metadaten nach den FAIR-Prinzipien, Datenmanagementplänen, Repositorien für Forschungsdaten, GO FAIR bis hin zu Persistenten Identifikatoren erstreckten sich die Themen.

Nach der dreijährigen Projektphase liegen bemerkenswerte Ergebnisse vor:

- Implementierung von österreichweit nutzbarer Infrastruktur für DOls an der Technischen Universität Wien

- Implementierung von österreichweit nutzbarer Infrastruktur für ORCID iDs durch die Gründung von ORCID Austria durch die Technische Universität Wien und die Universität Wien

- Verbreitung von Erkenntnissen zu verschiedenen Themen zum Forschungsdatenmanagement durch ca. 30 Veröffentlichungen aus den verschiedenen Arbeitspaketen

- Unterstützung der Einrichtung von institutionellen Repositorien durch die Veröffentlichung eines anwendbaren Prozesses zur Auswahl eines solchen

- Verankerung von Forschungsdatenmanagement an den Universitäten durch den Entwurf und/ oder der Einführung von FDM-Policies bei den Projektpartnern

- Beginn der Entwicklung von machine-actionable DMPs und eines passenden DMP Tools und damit verbundene Veröffentlichungen auf Github

- Weiterentwicklung eines Repositorienmanager-Netzwerks durch Workshops „Linking Open Science in Austria“ und „Certification Workshop on FAIR-aligned repositories in Austria" 
Durch den Austausch mit anderen Universitäten und den geschaffenen Kontakt zu den Forschenden konnten die Projektpartner zudem individuelle Handlungsfelder identifizieren, die zukünftig angegangen werden können. Das Projekt trug zur Sensibilisierung zum Thema Forschungsdatenmanagement auf verschiedenen Ebenen in den Universitäten bei. Die Evaluation des Projekts bei den beteiligten Projektpartnern zeigt, dass die Entwicklung in Richtung Open Science an Fahrt gewonnen hat. Allerdings werden die österreichischen Universitäten ohne weiteren Ausbau von Infrastruktur im nationalen und internationalen Wissenschaftswettbewerb nicht bestehen können. Einige der Themen, die uns zukünftig beschäftigen werden sind: Repositorien, Anlaufstellen zum FDM, PIDs, Zertifizierung von Repositorien und FAIR zertifizierte Infrastruktur, um den zukünftigen Anforderungen der Fördergeber zu genügen, EOSC als europaweite Infrastruktur für Forschende, Weiterentwicklung von Services in Zusammenarbeit mit Forschenden, domänenspezifische Metadatenmodelle, domänenspezifische Datenservices, Current Research Information Systems (CRIS), Research Impact Assessment, Research Promotion. Der Dank gilt allen Beteiligten, ohne deren Engagement der Fortschritt an den Universitäten nicht möglich gewesen wäre.

\section{Veröffentlichungen \& Präsentationen}

\subsection{Publikationen}

Im Projekt e-Infrastructures Austria Plus sind zahlreiche Publikationen rund um das Thema Open Science entstanden, die nach den FAIR-Prinzipien veröffentlicht wurden.

\section{Endbericht}

- Haselwanter, Thomas \& Thöricht, Heike (2020). e-Infrastructures Austria Plus Projektbericht 2017-2019. https://doi.org/10.25651/1.2020.0006

\section{Research Lifecycle}

- Kalová, Tereza (2019). Maßnahmen des Forschungsdatenmanagements an österreichischen Wissenschaftsorganisationen. https://doi. org/10.25651/1.2019.0005

- Katzmayr, Michael \& Seyffertitz, Thomas (2019). Leitfaden zur Erhebung zum Forschungsdatenmanagement. https://doi. org/10.25651/1.2019.0021 
- Katzmayr, Michael \& Seyffertitz, Thomas (2019). Forschungsdatenmanagement an der Wirtschaftsuniversität Wien. https://doi. org/10.25651/1.2019.0004

- Sánchez Solís, Barbara \& Stork, Christiane (2019). Forschungsdatenmanagement an der Technischen Universität Wien. https://doi. org/10.25651/1.2019.0012

\section{Policy zum Forschungsdatenmanagement}

- Policy zum Forschungsdatenmanagement der Universität für Musik und darstellende Kunst Wien. https://www.mdw.ac.at/upload/ MDWeb/forschungsfoerderung/downloads/FDM_Policy_mdw_ DE_20171128endR_MB-01.pdf

- Policy zum Forschungsdatenmanagement der Medizinischen Universität Wien. https://www.meduniwien.ac.at/web/fileadmin/content/ serviceeinrichtungen/itsc/it4science/Policy_fuer_ForschungsdatenManagement_v1.0.pdf

- Policy zum Forschungsdatenmanagement der Technischen Universität Wien. https://www.tuwien.at/forschung/fti-support/forschungsdaten/forschungsdatenmanagement/policy/

- Policy zum Forschungsdatenmanagement der Universität Graz. https:// static.uni-graz.at/fileadmin/strategische-entwicklung/Dateien/FDMPolicy_DE_FINAL_Layout.pdf

- Policy zum Forschungsdatenmanagement der Wirtschaftsuniversität Wien. https://www.wu.ac.at/bibliothek/services/forschungsdatenmanagement/wu-forschungsdatenmanagement-policy

\section{Datenmanagementpläne}

- Haselwanter, Thomas; Miksa, Tomas \& Thöricht, Heike (2019). Vergleich der DMP-Tools RDMO, DMPRoadmap und Data Steward Wizard. https://doi.org/10.25651/1.2019.0007

- Heider, Veronika; Raffetseder, Lena; Sánchez Solís, Barbara \& Ulrich, Xenia (2018). DMP Template for the Social Sciences (Version 1.0). https://doi.org/10.5281/zenodo.1291816

- Miksa, Thomas:

https://github.com/TomMiksa/DMPGenerator https://github.com/TomMiksa/digital_preservation_ex_1_2 https://github.com/TomMiksa/tu-dpue-lab2-ss18 https://github.com/TomMiksa/DigitalPreservation_2 https://github.com/TomMiksa/digitalpreservation-dmp-generator https://github.com/TomMiksa/DMPlanner 


\section{Institutionelle Repositorien}

- Haselwanter, Thomas \& Thöricht, Heike (2019). Anwendungsszenarien für Forschungsdatenrepositorien. https://doi. org/10.25651/1.2019.0008

- Haselwanter, Thomas \& Thöricht, Heike (2019). Erstellung von Persona zur Auswahl eines institutionellen Repositoriums für Forschungsdaten. https://doi.org/10.25651/1.2019.0010

- Haselwanter, Thomas \& Thöricht, Heike (2019). Der Ablageprozess von Forschungsdaten und was von Zenodo gelernt werden kann. https://doi.org/10.25651/1.2019.0006

- Haselwanter, Thomas \& Thöricht, Heike (2019). Klassifizierung von Forschungsdaten und Speichersysteme. https://doi. org/10.25651/1.2019.0009

\section{Metadaten}

- Bettel, Florian (2019). Austausch mit HRSM-Projekt „Portfolio/ Showroom - Making Art Resarch Accessible“. https://doi. org/10.25651/1.2019.0015

- Blumesberger, Susanne \& Zartl, Alexander (2019). Umgang mit Metadaten in Repositorien - eine österreichweite Umfrage. Zweite Folge. https://doi.org/10.25651/1.2019.0018

- Fiala, Sonja (2019). Kennzeichnung barrierefreier Dateien - eine Zusammenstellung am Beispiel MARC21 und MODS. https://doi. org/10.25651/1.2019.0014

- Fiala, Sonja \& Huggle, Christina (2019). Metadatenmapping - Die Gegenüberstellung verschiedener Metadatenschemata am Beispiel UWMETADATA>>MODS 3.6. https://doi.org/10.25651/1.2019.0013

- Gasteiner, Martin (2019). Interviews zu Forschungsdaten im Rahmen des Projekts E-Infrastructure Austria Plus. https://hdl.handle. net/11353/10.1008782

- Gründhammer, Veronika \& Sakabe, Yukiko (2019). Zum Umgang mit Metadaten an der ÖAW: Eine erste Annäherung. https://doi. org/10.25651/1.2019.0019

- Kalová, Tereza (2019). Metadaten für Forschungsdaten: Bedürfnisse und Anforderungen in den Naturwissenschaften. https://doi. org/10.25651/1.2019.0020

- Kennel, Patrik (2019). Sacherschließung von Forschungsdaten. https:// doi.org/10.25651/1.2019.0017

- Zartl, Alexander (2019). Automatische Übertragung von Metadaten in Videodateien. https://doi.org/10.25651/1.2019.0016 


\section{Persistente Idenfikatoren}

- Ferus, Andreas; Gstrein, Silvia; Hikl, Anna-Laetitia; Kaier, Christian; Kranewitter, Michael; Marín Arraiza, Paloma \& Mayer, Adelheid (2019). Institutionelle Muster-DOI-Policy. https://doi. org/10.25651/1.2019.0001

- Ferus, Andreas; Gstrein, Silvia; Hikl, Anna-Laetitia; Kaier, Christian; Kranewitter, Michael; Marín Arraiza, Paloma \& Mayer, Adelheid (2019). Institutional Model Policy for the Registration of Digital Object Identifiers. https://doi.org/10.25651/1.2019.0002

- Gstrein, Silvia \& Kaier, Christian (2017). DOI-Vergabe in Österreich: Szenarien. Internes Entscheidungspapier für Lenkungsausschuss.

- Heindl, Markus; Hikl, Anna-Laetitia; Kaier, Christian (2018). ORCID Austria Workshop (Wien, 24. Mai 2018). Mitteilungen der Vereinigung Österreichischer Bibliothekarinnen und Bibliothekare, 71(3-4), S. 468474. https://doi.org/10.31263/voebm.v71i3-4.2109

- ORCID Austria: https://www.tuwien.at/kooperationen/orcid/

\section{GO FAIR}

- Zweitägiger Workshop „Linking Open Science in Austria“ in Kooperation mit GOFAIR, BMBWF, OpenAIRE, RDA Austria und RDA Europe am 13. Juni 2019. https://linkingopenscience.univie.ac.at

- OpenAIRE-Blogpost „Connect and Get Connected - Linking Open Science in Austria". https://www.openaire.eu/blogs/connect-and-getconnected-linking-open-science-in-austria

- OpenAIRE, Workshop Series „Services to Support FAIR“. https://www. openaire.eu/workshops-series-services-to-support-fair-data-from-theory-to-implementation

\section{Exkurs}

- Marín Arraiza, Paloma (2019). ORCID: Member API oder Public API? https://doi.org/10.25651/1.2019.0011

\subsection{Workshops, Präsentationen und Informationsveranstaltungen}

- 14.11.2019 Paolo Budroni: Certification workshop on FAIR-aligned repositories in Austria, in Kooperation mit den Data Archiving and Networked Services, TU Wien Bibliothek.

- 12.09.2019 Thomas Haselwanter und Eva Ramminger: e-Infrastructures Austria Plus: Stand und Ergebnisse zum Projekt (34. Österreichischen Bibliothekartag 2019, Graz, 10.-13.09.2019). 
- 26.04.2019 Eva Ramminger: Vorstellung des Projekts e-Infrastructures Austria Plus (RepManNet-Tagung, UB Universität für Angewandte Kunst Wien).

- 24./25.04.2019 Paolo Budroni: Workshop Linking Open Science in Austria (Wien).

- 12.-15.06.2018 Thomas Haselwanter: e-Infrastructures Austria Plus Gemeinsam die Basis für RDM legen (107. Deutscher Bibliothekartag, Berlin).

- 07.06.2018 Eva Ramminger: e-Infrastructures Austria Plus. Statusbericht Juni 2018 (Sitzung des Forums Universitätsbibliotheken Österreichs (UBIFO), Wien).

- 24.05.2018 Markus Heindl, Anna-Laetitia Hikl, Christian Kaier: ORCID Austria Workshop (Wien).

- 08.03.2018 Eva Ramminger: e-Infrastructures Austria Plus. Statusbericht März 2018 (Sitzung des Forums Universitätsbibliotheken Österreichs (UBIFO), Wien).

- 21.-23.02.2018 Eva Ramminger: Forschungsdatenmanagement in der Praxis: Das Projekt e-Infrastructures Austria Plus (17. Österreichisches Online-Informationstreffen, 14. InetBib-Tagung, Wien).

- 23.11.2017 Thomas Haselwanter: e-Infrastructures Austria Plus (RDA Europe Workshop, Wien).

- 20.06.2017 Eva Ramminger: Research Data Management at Austrian Universities. The project „e-Infrastructures Austria Plus” (38th Annual IATUL (International Association of University Libraries) Conference, Bozen).

- 16.05.2017 Eva Ramminger: Statusbericht Mai 2017 zum Projekt e-infrastructures Austria Plus (Sitzung des Forums Universitätsbibliotheken Österreichs (UBIFO), Innsbruck).

- 18.11.2016 Eva Ramminger: Das Nachfolgeprojekt „e-Infrastructures Austria Plus“ (Generalversammlung „e-Infrastructures Austria“, Wien).

Mag. Thomas Haselwanter ORCID: https://orcid.org/0000-0001-9160-0180 Universität Innsbruck, Zentraler Informatikdienst E-Mail: thomas.haselwanter@uibk.ac.at 


\section{Literatur}

Adam, Beatrix \& Lindstädt, Birte (2019). Elektronische Laborbücher im Kontext von Forschungsdatenmanagement und guter wissenschaftlicher Praxis - ein Wegweiser für die Lebenswissenschaften : ELN-Wegweiser. https://doi.org/10.4126/FRL01-006415715

BARTOC. http://bartoc.org/

Bettel, Florian (2019). Austausch mit HRSM-Projekt „Portfolio/ Showroom Making ArtResarch Accessible“.https://doi.org/10.25651/1.2019.0015 Blumesberger, Susanne \& Zartl, Alexander (2019). Umgang mit Metadaten in Repositorien - eine österreichweite Umfrage. Zweite Folge. https:// doi.org/10.25651/1.2019.0018

Blumesberger, Susanne; Preza, Jose-Luis \& Zartl, Alexander (2016). Cluster I: Umgang mit Metadaten in Repositorien - Eine österreichweite Umfrage. https://hdl.handle.net/11353/10.441216

Blumesberger, Susanne \& Zartl, Alexander (2017). Umgang mit Metadaten in Repositorien - Eine österreichweite Umfrage. Mitteilungen der Vereinigung Österreichischer Bibliothekarinnen \& Bibliothekare, 70(2), S. 249-273. https://doi.org/10.31263/voebm.v70i2.1850

DANS. https://dans.knaw.nl/en

Dataverse. https://dataverse.org/

DCC, DMP Online - Funder Requirements. https://dmponline.dcc.ac.uk/ public_templates

DSpace. https://duraspace.org/dspace/

Elsevier. https://www.elsevier.com/

European Commission (2019). Orientations towards the first Strategic Plan for Horizon Europe. https://ec.europa.eu/info/sites/info/files/ research_and_innovation/strategy_on_research_and_innovation/documents/ec_rtd_orientations-he-strategic-plan_122019.pdf

Ex Libris. https://www.exlibrisgroup.com/de/

FAIRsharing. https://fairsharing.org/

Ferus, Andreas; Gstrein, Silvia; Hikl, Anna-Laetitia; Kaier, Christian; Kranewitter, Michael; Marín Arraiza, Paloma \& Mayer, Adelheid (2019). Institutionelle Muster-DOI-Policy. https://doi.org/10.25651/1.2019.0001

Ferus, Andreas; Gstrein, Silvia; Hikl, Anna-Laetitia; Kaier, Christian; Kranewitter, Michael; Marín Arraiza, Paloma \& Mayer, Adelheid (2019). Institutional Model Policy for the Registration of Digital Object Identifiers. https://doi.org/10.25651/1.2019.0002

Fiala, Sonja (2019). Kennzeichnung barrierefreier Dateien - eine Zusammenstellung am Beispiel MARC21 und MODS. https://doi. org/10.25651/1.2019.0014 
Fiala, Sonja \& Huggle, Christina (2019). Metadatenmapping - Die Gegenüberstellung verschiedener Metadatenschemata am Beispiel UWMETADATA >>MODS 3.6. https://doi.org/10.25651/1.2019.0013 figshare. https://figshare.com/

FWF, Datenmanagementplan (DMP) Vorlage - Guide. https:// www.fwf.ac.at/fileadmin/files/Dokumente/Open_Access/FWF_ DMPTemplate_d.pdf

Gasteiner, Martin (2019). Interviews zu Forschungsdaten im Rahmen des Projekts E-Infrastructure Austria Plus. https://hdl.handle. net/11353/10.1008782

GNU Lesser General Public License. http://www.gnu.org/licenses/Igpl3.0.html

Gründhammer, Veronika \& Sakabe, Yukiko (2019). Zum Umgang mit Metadaten an der ÖAW: Eine erste Annäherung. https://doi. org/10.25651/1.2019.0019

Gstrein, Silvia \& Kaier, Christian (2017). DOI-Vergabe in Österreich: Szenarien. Internes Entscheidungspapier für Lenkungsausschuss.

Haselwanter, Thomas; Miksa, Tomas \& Thöricht, Heike (2019). Vergleich der DMP-Tools RDMO, DMPRoadmap und Data Steward Wizard. https://doi.org/10.25651/1.2019.0007

Haselwanter, Thomas \& Thöricht, Heike (2020). e-Infrastructures Austria PlusProjektbericht2017-2019.https://doi.org/10.25651/1.2020.0006 Haselwanter, Thomas \& Thöricht, Heike (2019). Anwendungsszenarien für Forschungsdatenrepositorien. https://doi.org/10.25651/1.2019.0008 Haselwanter, Thomas \& Thöricht, Heike (2019). Erstellung von Persona zur Auswahl eines institutionellen Repositoriums für Forschungsdaten. https://doi.org/10.25651/1.2019.0010

Haselwanter, Thomas \& Thöricht, Heike (2019). Der Ablageprozess von Forschungsdaten und was von Zenodo gelernt werden kann. https:// doi.org/10.25651/1.2019.0006

Haselwanter, Thomas \& Thöricht, Heike (2019). Klassifizierung von Forschungsdaten und Speichersysteme. https://doi. org/10.25651/1.2019.0009

Heider, Veronika; Raffetseder, Lena; Sánchez Solís, Barbara \& Ulrich, Xenia (2018). DMP Template for the Social Sciences (Version 1.0). https:// doi.org/10.5281/zenodo.1291816

Heindl, Markus; Hikl, Anna-Laetitia; Kaier, Christian (2018). ORCID Austria Workshop (Wien, 24. Mai 2018). Mitteilungen der Vereinigung Österreichischer Bibliothekarinnen und Bibliothekare, 71(3-4), S. 468474. https://doi.org/10.31263/voebm.v71i3-4.2109 
INVENIO. https://invenio-software.org/

Kalová, Tereza (2019). Maßnahmen des Forschungsdatenmanagements an österreichischen Wissenschaftsorganisationen. https://doi. org/10.25651/1.2019.0005

Kalová, Tereza (2019). Metadaten für Forschungsdaten: Bedürfnisse und Anforderungen in den Naturwissenschaften. https://doi. org/10.25651/1.2019.0020

Katzmayr, Michael \& Seyffertitz, Thomas (2019). Leitfaden zur Erhebung zum Forschungsdatenmanagement. https://doi. org/10.25651/1.2019.0021

Katzmayr, Michael \& Seyffertitz, Thomas (2019). Forschungsdatenmanagement an der Wirtschaftsuniversität Wien. https://doi. org/10.25651/1.2019.0004

KDE. https://kde.org/

Kennel, Patrik (2019). Sacherschließung von Forschungsdaten. https:// doi.org/10.25651/1.2019.0017

LIBER DMP Catalogue. https://zenodo.org/communities/liber-dmp-cat Marín Arraiza, Paloma (2019). ORCID: Member API oder Public API? https://doi.org/10.25651/1.2019.0011

Medizinische Universität Wien, Policy zum Forschungsdatenmanagement der Medizinischen Universität Wien. https://www.meduniwien.ac.at/ web/fileadmin/content/serviceeinrichtungen/itsc/it4science/Policy_ fuer_Forschungsdaten-Management_v1.0.pdf

Miksa, Thomas: https://github.com/TomMiksa/DMPGenerator

Miksa, Thomas: https://github.com/TomMiksa/digital_preservation_ex_1_2 Miksa, Thomas: https://github.com/TomMiksa/tu-dpue-lab2-ss18

Miksa, Thomas: https://github.com/TomMiksa/DigitalPreservation_2

Miksa, Thomas: https://github.com/TomMiksa/digitalpreservation-dmpgenerator

Miksa, Thomas: https://github.com/TomMiksa/DMPlanner

OpenAIRE, „Connect and Get Connected - Linking Open Science in Austria“. https://www.openaire.eu/blogs/connect-and-get-connected-linking-open-science-in-austria

OpenAIRE, Workshop Series „Services to Support FAIR“. https://www. openaire.eu/workshops-series-services-to-support-fair-data-from-theory-to-implementation

ORCID Austria. https://www.tuwien.at/kooperationen/orcid/

Portfolio/Showroom. https://portfolio-showroom.ac.at/

RDA, DMP Common Standards WG. https://www.rd-alliance.org/ groups/dmp-common-standards-wg 
Sánchez Solís, Barbara \& Stork, Christiane (2019). Forschungsdatenmanagement an der Technischen Universität Wien. https://doi. org/10.25651/1.2019.0012

Science Europe, Practical Guide to the International Alignment of Research Data Management. https://www.scienceeurope.org/our-resources/ practical-guide-to-the-international-alignment-of-research-data-management/

Technische Universität Wien, Policy zum Forschungsdatenmanagement der Technischen Universität Wien. https://www.tuwien.at/forschung/ fti-support/forschungsdaten/forschungsdatenmanagement/policy/

TIND. https://tind.io/

UK Data Service, Research data lifecycle. https://www.ukdataservice. ac.uk/manage-data/lifecycle.aspx

Universität für Musik und darstellende Kunst Wien, Policy zum Forschungsdatenmanagement der Universität für Musik und darstellende Kunst Wien. https://www.mdw.ac.at/upload/MDWeb/forschungsfoerderung/downloads/FDM_Policy_mdw_DE_20171128endR_MB01.pdf

Universität Graz, Policy zum Forschungsdatenmanagement der Universität Graz. https://static.uni-graz.at/fileadmin/strategische-entwicklung/ Dateien/FDM-Policy_DE_FINAL_Layout.pdf

University of Central Florida, Research Lifecycle at UCF. https://stars.library.ucf.edu/ucfrlc/

Wirtschaftsuniversität Wien, Policy zum Forschungsdatenmanagement der Wirtschaftsuniversität Wien. https://www.wu.ac.at/bibliothek/ services/forschungsdatenmanagement/wu-forschungsdatenmanagement-policy

Workshop „Linking Open Science in Austria“ in Kooperation mit GOFAIR, BMBWF, OpenAIRE, RDA Austria und RDA Europe am 13. Juni 2019. https://linkingopenscience.univie.ac.at

Zartl, Alexander (2019). Automatische Übertragung von Metadaten in Videodateien. https://doi.org/10.25651/1.2019.0016

1 https://ec.europa.eu/info/sites/info/files/research_and_innovation/ strategy_on_research_and_innovation/documents/ec_rtd_orientations-he-strategic-plan_122019.pdf

2 An dieser Stelle wird auf den ELN Wegweiser des ZB MED - Informationszentrums Lebenswissenschaften hingewiesen, der im August 2019 veröffentlicht wurde. https://doi.org/10.4126/FRL01-006415715

3 https://doi.org/10.25651/1.2020.0006-001 
4 Katzmayr, Michael \& Seyffertitz, Thomas (2019). Leitfaden zur Erhebung zum Forschungsdatenmanagement. https://doi. org/10.25651/1.2019.0021

5 https://stars.library.ucf.edu/ucfrlc/

6 https://www.ukdataservice.ac.uk/manage-data/lifecycle.aspx

7 Die verwendeten Icons entstammen dem Projekt https://kde.org/ und sind frei verwendbar unter den Bedingungen der GNU LGPLv3 (http:// www.gnu.org/copyleft/lesser.html)

8 https://doi.org/10.25651/1.2020.0006-002

9 Mehr Informationen zum Leaders Activating Research Networks (LEARN) Projekt sind unter http://learn-rdm.eu/en/about zu finden. Eine Musterpolicy zum FDM wurde veröffentlicht (vgl. Leaders Activating Research Networks 2017a)

10 https://www.mdw.ac.at/forschungsförderung/?Pageld=4264

11 https://www. meduniwien.ac.at/web/rechtliches/policy-fuer-forschungsdatenmanagement/

12 https://www.tuwien.at/forschung/fti-support/forschungsdaten/forschungsdatenmanagement/policy/

13 https://strategische-entwicklung.uni-graz.at/de/strategieumsetzung/ publikationsstrategie-open-access/

14 https://www.wu.ac.at/bibliothek/services/forschungsdatenmanagement/wu-forschungsdatenmanagement-policy

15 https://www.forschungsdaten.info/fdm-im-deutschsprachigen-raum/ oesterreich/fdm-policies/

16 Università degli Studi di Milano (2017)

17 Università di Padova (2018)

18 https://doi.org/10.25651/1.2020.0006-003

19 FWF Datenmanagementplan (DMP) Vorlage - Guide. https:// www.fwf.ac.at/fileadmin/files/Dokumente/Open_Access/FWF_ DMPTemplate_d.pdf

20 Practical Guide to the International Alignment of Research Data Management. https://www.scienceeurope.org/wp-content/uploads/2018/12/SE_RDM_Practical_Guide_Final.pdf

21 siehe Katalog unter https://zenodo.org/communities/liber-dmp-cat/

22 siehe Katalog unter https://dmponline.dcc.ac.uk/public_templates

23 Heider, Veronika; Raffetseder, Lena; Sanchez Solis, Barbara \& Ulrich, Xenia (2018). DMP Template for the Social Sciences (Version 1.0). Zenodo. https://doi.org/10.5281/zenodo.1291816

24 genauere Informationen über den Piloten finden sich im Endbericht, Kapitel 4.3. https://doi.org/10.25651/1.2020.0006 
25 Miksa, Tomasz et al. (2019)

26 https://www.rd-alliance.org/groups/dmp-common-standards-wg

27 https://doi.org/10.25651/1.2020.0006-004

28 FWF (2019b) et al., Rohsmann, Katarina (2016)

29 Erstellung von Persona zur Auswahl eines institutionellen Repositoriums für Forschungsdaten. https://doi.org/10.25651/1.2019.0010

$30 \mathrm{vgl}$. Repository Platforms for Research Data Interest Group of the Research Data Alliance (2016)

31 Zhang, Tao et al.(2013)

32 CoreTrustSeal (2018)

33 https://invenio-software.org/

34 https://dataverse.org/

35 https://duraspace.org/dspace/

36 Wikipedia contributors (23. September 2019)

37 https://tind.io/

38 https://figshare.com/

39 Figshare (o. A.)

40 Wikipedia contributors (4. Oktober 2019)

41 https://www.elsevier.com/

42 Elsevier (o. A.)

43 https://www.exlibrisgroup.com/de/

44 ExLibris (o. A.)

45 https://doi.org/10.25651/1.2020.0006-007

46 Blumesberger, Susanne \& Zartl, Alexander (2017). Umgang mit Metadaten in Repositorien - Eine österreichweite Umfrage. Mitteilungen der Vereinigung Österreichischer Bibliothekarinnen \& Bibliothekare, 70(2), S. 249-273. https://doi.org/10.31263/voebm.v70i2.1850

47 https://doi.org/10.25651/1.2019.0019

48 http://phaidra.univie.ac.at/o:441216

49 https://doi.org/10.25651/1.2019.0020

50 https://doi.org/10.25651/1.2019.0013

51 https://doi.org/10.25651/1.2019.0016

52 https://doi.org/10.25651/1.2019.0017

53 http://bartoc.org/

54 https://fairsharing.org/

55 https://phaidra.univie.ac.at/o:1008782

56 https://doi.org/10.25651/1.2019.0015

57 vgl. https://portfolio-showroom.ac.at/

58 https://doi.org/10.25651/1.2020.0006-006

59 Wikipedia contributors (20. September 2019) 
60 ORCID (o. A.)

61 Heindl, Markus et al. (2018)

62 https://www.tuwien.at/kooperationen/orcid/en/home/

63 https://doi.org/10.25651/1.2020.0006-007

64 Mehr Informationen zu DANS sind auf der Website https://dans.knaw. $\mathrm{nl} /$ en verfügbar.

65 Dieser Workshop fand nach Redaktionsschluss des Berichts statt. 